\title{
Anderson-Hubbard model in infinite dimensions
}

\author{
M. Ulmke* \\ Institut für Festkörperforschung, Forschungszentrum Jülich, 52425 Jülich, Federal Republic of Germany \\ V. Janiš ${ }^{\dagger}$ and D. Vollhardt \\ Institut für Theoretische Physik C, Technische Hochschule Aachen, 52056 Aachen, Federal Republic of Germany
}

(Received 13 September 1994)

\begin{abstract}
We present a detailed, quantitative study of the competition between interaction- and disorderinduced effects in electronic systems. For this the Hubbard model with diagonal disorder (AndersonHubbard model) is investigated analytically and numerically in the limit of infinite spatial dimensions, i.e., within a dynamical mean-field theory, at half-filling. Numerical results are obtained for three different disorder distributions by employing quantum Monte Carlo techniques, which provide an explicit finite-temperature solution of the model in this limit. The magnetic phase diagram is constructed from the zeros of the inverse averaged staggered susceptibility. We find that at low enough temperatures and sufficiently strong interaction there always exists a phase with antiferromagnetic long-range order. A strong coupling anomaly, i.e., an increase of the Néel temperature for increasing disorder, is discovered. An explicit explanation is given, which shows that in the case of diagonal disorder this is a generic effect. The existence of metal-insulator transitions is studied by evaluating the averaged compressibility both in the paramagnetic and antiferromagnetic phases. A rich transition scenario, involving metal-insulator and magnetic transitions, is found and its dependence on the choice of the disorder distribution is discussed.
\end{abstract}

\section{INTRODUCTION}

The investigation of interacting electronic systems is one of the most intriguing, albeit difficult, subjects in condensed matter physics. The same is true for the study of disordered, non-interacting electrons. In view of the theoretical complexity of the two problems taken separately, it is understandable that their combination, i.e., the simultaneous presence of randomness and interactions, as found in many real systems (e.g., doped semiconductors near the metal-insulator transition, high- $T_{c}$ superconducting materials close to $T_{c}$, etc.), leads to new, fundamental questions to which only few secured answers are known. This is all the more true when the interaction and/or the disorder is strong, since there exist hardly any tractable, and at the same time controlled, theoretical method of investigation in this limit.

An important starting point for the investigation of interacting, disordered systems was the field-theoretic approach developed for the treatment of noninteracting electrons, i.e., the scaling theory of Anderson localization. ${ }^{1-3}$ A generalization of this theory to finite interactions by Finkelshtein ${ }^{4}$ provided essential new insight. ${ }^{5}$ However, the appearance of local magnetic moments in the renormalization group treatment discovered by him $^{6}$ and Castellani et al. ${ }^{7}$ turned out to be a fundamental obstacle for the study of the metal-insulator transition (MIT) itself. ${ }^{8,9}$ A microscopic origin of this instability towards the formation of localized moments can already be traced within Hartree-Fock theory for the disordered Hubbard model ("Anderson-Hubbard model") with off-diagonal disorder. ${ }^{10,11}$ The results indicate that the above renormalization-group approach, as well as is starting point, i.e., Anderson-localization, are not appropriate when it comes to the investigation of threedimensional disordered electrons with intermediate or strong interactions close to half-filling $(n \simeq 1)$. After all, a MIT occurs even without disorder in this case, and the lattice periodicity becomes essential. A nonperturbative and qualitatively quite different, but essentially uncontrolled, renormalization technique by which disorder and interactions can be treated on equal footing is the real space renormalization-group approach. It was first applied by $\mathrm{Ma}^{12}$ and recently used by $\mathrm{Yi}$ et al. ${ }^{13}$ to investigate the Anderson-Hubbard model with diagonal disorder, i.e., random on-site energies, in dimensions $d=1,3$ at $n=1$ and in $d=2$ for $n \lesssim 1$, respectively. However, in these investigations the formation of antiferromagnetic long-range order (AFLRO), which will always set in at large enough repulsion (at least at $n=1$ ) was not considered. The strong-coupling limit of this model was studied by Zimanyi and Abrahams ${ }^{14}$ using a slaveboson formulation of the corresponding $t-J$ model. The considerably simpler, but still highly nontrivial, case of disordered, spinless fermions in $d=1$ was also addressed recently. ${ }^{15,16}$

To obtain a global picture of the properties of interacting, disordered systems it is desirable to know the solution of a simple, microscopic model which is valid for all input parameters (interaction, disorder, temperature, band filling). Since exact solutions are not available in $d=2,3$ one would like to construct, at least, a thermodynamically consistent mean-field theory that is valid also at strong coupling. Such a (nonperturba- 
tive) approximation is provided by the exact solution of a model in $d=\infty$. It is now known that even in the limit $d \rightarrow \infty$ (Refs. 17 and 18) the Hubbard interaction remains dynamical ${ }^{19}$ and leads to a highly nontrivial singlesite problem ${ }^{20-27}$ with infinitely many coupled quantum degrees of freedom. This problem is, in fact, equivalent with an Anderson impurity model complemented by a self-consistency condition ${ }^{21,24,27}$ and is thus amenable to numerical investigations within a finite-temperature quantum Monte Carlo approach. ${ }^{28}$ In the absence of disorder this technique was already used by several groups to investigate the magnetic phase diagram, ${ }^{24,25,29}$ the Mott-Hubbard transition, ${ }^{30,32,29}$ transport properties, ${ }^{33}$ and lately also superconductivity in a two-band version ${ }^{34}$ of the Hubbard model in $d=\infty$. These investigations were also extended to the periodic Anderson model ${ }^{35}$ and the Holstein model. ${ }^{36}$

The dynamical mean-field theory of interacting electrons obtained in the limit $d=\infty$ has many appealing features; however, it also has its limitations, which are due to the purely local nature of the theory. One effect which cannot be described within the $d=\infty$ meanfield theory of disordered electrons is the phenomenon of Anderson localization. That is, the electrons in infinite dimensions are delocalized for arbitrary strength of the disorder. The absence of localized states is caused by the above mentioned single-site dependence, i.e., by the fact that momentum dependence enters the physical quantities only through the dispersion relation, whereby vertex corrections to the conductivity vanish identically. ${ }^{37}$ In fact, it was shown ${ }^{38,39}$ that for vanishing interaction, when the Anderson-Hubbard model reduces to the Anderson disorder model, the well-known and much-used "coherent potential approximation (CPA)," 40,41 which does not describe Anderson localization, yields the exact solution in $d=\infty$. Nevertheless it should be stressed that even in the absence of Anderson localization the presence of disorder can have a significant, nontrivial effect on the properties of an interacting system. First results of a quantum Monte Carlo study of the competition between disorder and interaction in the Anderson-Hubbard in $d=\infty$ with diagonal "binary-alloy" disorder were recently reported by us. ${ }^{42}$ The importance of separable, off-diagonal disorder in the formation of local moments and the Mott transition was investigated by Dobrosavljević and Kotliar. ${ }^{43}$

A good impression of how complicated the phase diagram and the dynamical properties of interacting electrons with and without disorder are may be inferred from the results for spinless electron in $d=\infty .{ }^{44,45}$ The fact that the interactions reduce to their Hartree-contribution in this case, ${ }^{19}$ i.e., are no longer dynamical, does not at all imply that the properties of the model become trivial - quite the contrary. Many details of this model can be obtained analytically in $d=\infty$, thereby providing valuable insight into the effects of interactions, ${ }^{45}$ the interplay between interaction and disorder, ${ }^{44}$ and the $d=\infty$ limit itself. $^{46}$

The correlations caused by interactions between electrons and by the scattering of electrons in a disordered medium, respectively, can be very different; indeed, they may lead to opposite effects. For example, even an arbitrarily weak repulsive Hubbard interaction between electrons on a bipartite lattice will induce AFLRO when the band filling is sufficiently close to $n=1$ (at least in dimensions $d>2$ ). By contrast, it is the very nature of a random potential to oppose order. Hence interactions and disorder are expected to compete with each other. This conclusion may appear self-evident and almost trivial but is really only based on a simple-minded superposition of the two individual physical effects (which may, in fact, be quite correct for weak interactions and disorder). When the interaction and/or disorder is strong, however, this picture looses its basis. In general, the mutual interplay of those two effects can be expected to lead to novel, nonperturbative, quantum-mechanical many-body phenomena, e.g., new phases, which have no analog in nonrandom, interacting or disordered, noninteracting systems.

In view of the interplay between disorder and interaction there are a number of simple questions whose answers will be nontrivial. For example:

(1) Is there a finite disorder strength above which AFLRO ceases to exist? (After all, the fluctuations of the ensembles around the average value $n=1$ induced by the disorder might become so large that AFLRO is made impossible.)

(2) Does disorder always reduce the critical temperature for AFLRO, as one might expect intuitively?

(3) Do interactions always drive a system away from metallic behavior into an insulating state?

(4) How important is the choice of disorder distribution on the results?

These are questions which need to be answered by means of controlled investigations of a well-defined microscopic model. It is our intention in this paper to provide explicit, numerically exact answers to the above questions within the dynamical mean-field theory obtained in the limit of high spatial dimensions. A preliminary account was presented in Ref. 42. The paper is structured as follows. In Sec. II, we introduce the model and discuss the form of the corresponding averaged free energy in $d=\infty$. In Sec. III, we calculate correlation functions, i.e., the averaged compressibility and staggered susceptibility, to determine the thermodynamic stability of various phases. The details of the model (input parameters, disorder distribution, etc.) are specified and the numerical procedure is discussed in Sec. IV. This leads to the construction of the magnetic phase diagram in Sec. V. In Sec. VI, the results on magnetic phase transitions are then complemented by those on metal-insulator transitions. A discussion of the combined phase transition scenario closes the presentation.

\section{MODEL AND AVERAGED FREE ENERGY IN $D=\infty$}

The simplest microscopic Hamiltonian for conduction electrons interacting via the local Hubbard interaction in a disordered system is the so-called Anderson-Hubbard model, which can be written as 


$$
\hat{H}=\sum_{i, j, \sigma} t_{i j} \hat{c}_{i \sigma}^{+} \hat{c}_{j \sigma}+U \sum_{i} \hat{n}_{i \uparrow} \hat{n}_{i \downarrow}+\sum_{i, \sigma}\left(\epsilon_{i}-\mu_{\sigma}\right) \hat{n}_{i \sigma}
$$

Here, $\hat{c}_{i \sigma}^{+}\left(\hat{c}_{i \sigma}\right)$ create (annihilate) a $\sigma$ electron, on site $i$, with $\hat{n}_{i \sigma}=\hat{c}_{i \sigma}^{+} \hat{c}_{i \sigma}$ and $\mu_{\sigma}$ is the chemical potential of the $\sigma$ electrons. In general, both the hopping amplitude $t_{i j}$ and the atomic energies $\epsilon_{i}$ in (1) depend on the configuration of the atoms distributed on the lattice. In this paper we restrict ourselves to the case of uncorrelated, diagonal disorder. Hence, we assume the hopping elements to be independent of the randomness (with $t_{i j}=-t$ for nearest-neighbor sites and $t_{i j}=0$ otherwise), while the atomic potentials $\epsilon_{i}$ are chosen as random; thus, shortrange order is neglected. The Hamiltonian under investigation is then given by

$$
\hat{H}=\hat{H}_{\mathrm{Hub}}+\sum_{i, \sigma}\left(\epsilon_{i}-\mu_{\sigma}\right) \hat{n}_{i \sigma}
$$

where $\hat{H}_{\text {Hub }}$ is the usual Hubbard model,

$$
\hat{H}_{\mathrm{Hub}}=-\frac{t^{*}}{\sqrt{Z}} \sum_{\langle i j\rangle, \sigma} \hat{c}_{i \sigma}^{+} \hat{c}_{j \sigma}+U \sum_{i} \hat{n}_{i \uparrow} \hat{n}_{i \downarrow} .
$$

Here, the hopping matrix element was written as ${ }^{17}$

$$
t=t^{*} / \sqrt{Z}, t^{*}=\mathrm{const},
$$

with $Z$ as the number of nearest neighbors on the lattice (e.g., $Z=2 d$ on hypercubic lattices in $d$ dimensions). This scaling ensures that (3) remains nontrivial even in the limit $Z \rightarrow \infty$. We can then write the averaged full energy (grand potential) as

$$
\Omega_{\mathrm{av}}=-\beta^{-1}\langle(\ln \operatorname{Tr} \exp (-\beta \hat{H}))\rangle_{\mathrm{av}},
$$

where $\beta=1 / k_{B} T$. Here, the average over the disorder is defined as usual as

$$
\langle X\rangle_{\mathrm{av}}=\prod_{i}\left[\int_{-\infty}^{\infty} d \epsilon_{i} P\left(\epsilon_{i}\right)\right] X\left(\epsilon_{1}, \ldots, \epsilon_{L}\right),
$$

where $L$ is the number of lattice sites, and $P\left(\epsilon_{i}\right)$ is the distribution of the random potentials $\epsilon_{i}$. The explicit form of the function $P\left(\epsilon_{i}\right)$ will be specified later.

In realistic dimensions $(d=2,3)$, it is impossible to perform the trace over all fermionic states in (2) exactly. However, in the limit of infinite coordination number or spatial dimensions, significant simplifications occur in the evaluation of thermodynamic properties. Two of us recently showed ${ }^{39}$ that the averaged grand potential of the disordered Hubbard model in $d=\infty$ can be reduced to an expression, where the averaging is performed only on a single site. The explicit expression for the grand potential in the paramagnetic phase is given by

$$
\begin{aligned}
\Omega_{\mathrm{av}} / L= & -\beta^{-1} \sum_{\sigma n} \int_{-\infty}^{\infty} d E N(E) \ln \left[i \omega_{n}+\mu_{\sigma}\right. \\
& \left.-\Sigma_{\sigma n}-E\right] \\
& +\beta^{-1} \sum_{\sigma n} \ln G_{\sigma n}^{-1}-\beta^{-1}\left\langle\ln \mathcal{Z}\left\{\mathcal{G}^{-1}, \epsilon_{i}\right\}\right\rangle_{\mathrm{av}}
\end{aligned}
$$

where $N(E)$ is the density of states of noninteracting electrons, $\omega_{n}=(2 n+1) \pi \beta^{-1}$ are Matsubara frequen- cies, and the generalized atomic partition function $\mathcal{Z}$ is represented by a Grassmann functional integral,

$$
\mathcal{Z}\left\{\mathcal{G}^{-1}, \epsilon_{i}\right\}=\int \mathcal{D} \psi \mathcal{D} \psi^{*} e^{\mathcal{A}}
$$

with the local action

$$
\begin{aligned}
\mathcal{A}= & {\left[\sum_{\sigma, n} \psi_{\sigma n}^{*}\left(\mathcal{G}_{\sigma n}^{-1}-\epsilon_{i}\right) \psi_{\sigma n}\right.} \\
& \left.-U \int_{0}^{\beta} d \tau \psi_{\uparrow}^{*}(\tau) \psi_{\uparrow}(\tau) \psi_{\downarrow}^{*}(\tau) \psi_{\downarrow}(\tau)\right] .
\end{aligned}
$$

Here, $\Psi_{\sigma}(\tau)$ are Grassmann variables that depend on the imaginary time $\tau$, and $\Psi_{\sigma n}$ are their Fourier transforms into $\omega_{n}$ space. Only the random potentials $\epsilon_{i}$ are configuration dependent. Furthermore,

$$
\mathcal{G}_{\sigma n}^{-1}=G_{\sigma n}^{-1}+\Sigma_{\sigma n}
$$

is an effective local propagator of the electrons. The grand potential (6) is a functional of the complex quantities $G_{\sigma n}$ and $\Sigma_{\sigma n}$, which at this point enter as variational parameters. That is, the physical values of $G_{\sigma n}$ and $\Sigma_{\sigma n}$ (namely, the local Green function, $G_{\sigma n} \equiv G_{i i, \sigma n}$, and the self-energy, $\Sigma_{\sigma n} \equiv \Sigma_{i i, \sigma n}$, of the electrons, respectively) correspond to those for which (5) is stationary. ${ }^{22,47}$

Each term of $\Omega_{\mathrm{av}}$ in (6) has a clear mean-field interpretation, which allows us to outline a simple construction of $\Omega_{\mathrm{av}}$ on a mean-field level. ${ }^{39,18}$ We first introduce a homogeneous, ${ }^{48}$ effective, energy-dependent potential $\Sigma_{\sigma n}$, which is defined in such a way that in the thermodynamic limit the system of disordered, interacting electrons is equivalently described by a system of noninteracting electrons in the potential $\Sigma_{\sigma n}$. The free energy $\Omega_{\mathrm{av}}\left\{\Sigma_{\sigma n}\right\}$ is now constructed as follows: ${ }^{49}$ (i) we start with the free energy of noninteracting electrons in the potential $\Sigma_{\sigma n}$ [first term in (6)]; (ii) then we remove the potential $\Sigma_{\sigma n}$ from site $i$, i.e., subtract its energy contribution [second term in (6)]; and (iii) replace it by the potential $\hat{v}_{i \sigma}=\frac{1}{2} U \hat{n}_{i-\sigma}+\epsilon_{i}-\mu_{\sigma}$, and finally average over $\epsilon_{i}$ [last term in (6)]. ${ }^{50}$ To determine the potential $\Sigma_{\sigma n}$, we demand that the free energy obtained by this construction be stationary under variation of $\Sigma_{\sigma n}$, i.e., $\partial \Omega_{\mathrm{av}} / \partial \Sigma_{\sigma n}=0$. This is a self-consistent equation for $\Sigma_{\sigma n}$. The major advantage of the mean-field grand potential (6) is the property that the variational parameters are not explicitly configuration dependent, ${ }^{39}$ i.e., they are determined only by averaged quantities which result from the averaging in (6). Since the averaging is local, the disorder only leads to local correlations on the same site. Different lattice sites are effectively decoupled and all the information about the surrounding sites is contained in the effective potential $\Sigma_{\sigma n}$. Indeed, $\Sigma_{\sigma n}$ is a generalization of the "coherent potential" known from the theory of random alloys. ${ }^{40,41}$ This may be seen as follows: ${ }^{18,51}$ if we neglect the Hubbard interaction $U$, the Grassmann functional integral (7a) becomes Gaussian and can be performed exactly, leading to 


$$
\begin{aligned}
\Omega_{\mathrm{av}}(U=0) / L= & -\beta^{-1} \sum_{\sigma, n}\left\{\int _ { - \infty } ^ { \infty } d E N ( E ) \operatorname { l n } \left[i \omega_{n}+\mu_{\sigma}\right.\right. \\
& \left.-\Sigma_{\sigma n}-E\right]+\left\langle\operatorname { l n } \left[ 1+G_{\sigma n}\left(\Sigma_{\sigma n}\right.\right.\right. \\
& \left.\left.\left.\left.-\epsilon_{i}\right)\right]\right\rangle_{\mathrm{av}}\right\}
\end{aligned}
$$

This is precisely the grand potential of a random alloy with noninteracting electrons within CPA, with $\Sigma_{\sigma n}$ as the coherent potential expressed in Matsubara frequencies. ${ }^{41,51}$ Hence (6) can be understood as a fieldtheoretic generalization of the CPA to random alloys with interacting electrons, which becomes exact for $Z \rightarrow \infty$.

It should be noted that the averaged grand potential (6) does not contain the complete information about the equilibrium physics of random systems. ${ }^{39}$ For example, averaged products of Green functions contain new, nontrivial correlations and cannot be derived from (6). The averaged grand potential does, however, carry the full information about the equilibrium thermodynamics of the model. We concentrate in this paper exclusively on the thermodynamics of the Anderson-Hubbard model.

It is well known that, for large enough $U$, the Hubbard model itself leads to AFLRO at (or even close to) halffilling. This type of long-range order must, therefore, be considered even in the presence of disorder. For the sake of simplicity, we investigate only AFLRO on bipartite lattices. In this case, the breaking of symmetry is caused by a staggered magnetic field $h_{s}$ with $\left(+h_{s}\right)$ on $A$ and $\left(-h_{s}\right)$ on $B$ sites. To be able to study this type of order quantitatively, we extend the averaged grand potential (6) to the symmetry-broken phase. The averaged grand potential of a general antiferromagnetic solution has the form

$$
\begin{aligned}
\Omega_{\mathrm{av}} / L= & -\frac{1}{2 \beta}\left\{\sum _ { \sigma , n } \int _ { - \infty } ^ { \infty } d E N ( E ) \operatorname { l n } \left[\left(i \omega_{n}+\mu_{A \sigma}\right.\right.\right. \\
& \left.\left.-\Sigma_{A \sigma n}\right)\left(i \omega_{n}+\mu_{B \sigma}-\Sigma_{B \sigma n}\right)-E^{2}\right] \\
& \left.-\sum_{\sigma, n} \sum_{\gamma=A, B} \ln G_{\gamma \sigma n}^{-1}+\sum_{\gamma=A, B}\left\langle\ln \mathcal{Z}_{\gamma}\right\rangle_{\mathrm{av}}\right\},
\end{aligned}
$$

where the index $\gamma=(A, B) \equiv(+1,-1)$ corresponds to sublattices $A, B$, respectively, and $\mu_{\gamma \sigma}=\mu+\sigma\left(\gamma h_{s}+h\right)$, with $h$ as a magnetic field. The local partition function $\mathcal{Z}_{\gamma}$ in (10) is given by (7) with the replacements $\psi_{\sigma n} \rightarrow$ $\psi_{\gamma \sigma n}, \psi_{\sigma}(\tau) \rightarrow \psi_{\gamma \sigma}(\tau), G_{\sigma n}^{-1} \rightarrow G_{\gamma \sigma n}^{-1}$, etc., and integration over all Grassmann fields, i.e., $\mathcal{Z}_{\gamma}=\int \mathcal{D} \psi \mathcal{D} \psi^{*} e^{\mathcal{A}_{\gamma}}$, with

$$
\begin{aligned}
\mathcal{A}_{\gamma}= & \sum_{\sigma n} \psi_{\gamma \sigma n}^{*}\left(G_{\gamma \sigma n}^{-1}+\Sigma_{\gamma \sigma n}-\epsilon_{i}\right) \psi_{\gamma \sigma n} \\
& -U \int_{0}^{\beta} d \tau \psi_{\gamma \uparrow}^{*}(\tau) \psi_{\gamma \uparrow}(\tau) \psi_{\gamma \downarrow}^{*}(\tau) \psi_{\gamma \downarrow}(\tau) .
\end{aligned}
$$

The full partition function is given by $\mathcal{Z}=\mathcal{Z}_{A} \mathcal{Z}_{B}$. As described above the physical values of $G_{\gamma \sigma n}$ and $\Sigma_{\gamma \sigma n}$ in (10) are then found from the stationarity conditions,

$$
\delta \Omega_{\mathrm{av}} / \delta G_{\gamma \sigma n}=0, \delta \Omega_{\mathrm{av}} / \delta \Sigma_{\gamma \sigma n}=0 .
$$

In the following, we will set $h=0$ in (10) and exclude the existence of ferrimagnetism. In this case the average number of electrons on sublattices $A$ and $B$ are equal. Then there appears a new symmetry: quantities with indices $\gamma, \sigma$ and $-\gamma,-\sigma$ coincide. Hence, the double index $\gamma, \sigma$ can be combined into a single one, $\alpha=(+,-)$, with $\alpha=+$ for $A \uparrow=B \downarrow$ and $\alpha=-$ for $A \downarrow=B \uparrow$. Thereby the number of independent parameters is reduced. Equation (12) then yields two coupled sets of self-consistent equations for $G_{\alpha n}$ and $\Sigma_{\alpha n}$,

$$
\begin{aligned}
G_{\alpha n} & =\int_{-\infty}^{\infty} d E \frac{N(E)}{i \omega_{n}+\mu_{\alpha}-\Sigma_{\alpha n}-E^{2} /\left(i \omega_{n}+\mu_{-\alpha}-\Sigma_{-\alpha n}\right)} \\
G_{\alpha n} & =-\int_{0}^{\beta} d \tau e^{i \omega_{n} \tau}\left\langle\left\langle\psi_{\alpha}(\tau) \psi_{\alpha}^{*}(0)\right\rangle_{T}\right\rangle_{\mathrm{av}}
\end{aligned}
$$

where $\mu_{\alpha}=\mu+\alpha h_{s}$. Here \langle\rangle$_{T}$ represents the statistical average with the local action as

$$
\langle O\rangle_{T}=\frac{1}{\mathcal{Z}_{+} \mathcal{Z}_{-}} \int \mathcal{D} \psi \mathcal{D} \psi^{*} O e^{\mathcal{A}_{+}+\mathcal{A}_{-}} .
$$

Note that (13a) merely expresses the fact that $G_{\alpha n}$ is the local element of the full Green function, i.e., is an explicit function of the self energy $\Sigma_{\alpha n}$. Only Eq. (13b) describes the actual dynamics of the system, determining the self-energy $\Sigma_{\alpha n}$ as a function of the input parameters $T, \mu, U$ and the disorder distribution. Equation (13) fully determine the one-particle properties of the AndersonHubbard model in $d=\infty$.

\section{CORRELATION FUNCTIONS AND THERMODYNAMIC STABILITY}

To decide about the stability of a particular solution of (13), we have to evaluate averaged two-particle correlation functions or susceptibilities. Two such quantities are of particular interest in the Anderson-Hubbard model: the averaged staggered magnetic susceptibility $\chi_{\mathrm{av}}^{\mathrm{stag}} \equiv \chi_{\mathrm{AF}}$ and the averaged compressibility $\kappa_{\mathrm{av}}$. The former decides about the instability of the paramagnetic phase with respect to AFLRO, while the latter not only contains the information about a possible instability with respect to phase separation $\left(\kappa_{\mathrm{av}} \rightarrow \infty\right)$, but also provides a thermodynamic criterion for a solution to be insulating 
$\left(\kappa_{\mathrm{av}} \rightarrow 0\right)$. Quite generally susceptibilities can be obtained from the second derivative of the averaged grand potential $\Omega_{\mathrm{av}}$ with respect to some variable $x$ as

$$
X_{\mathrm{av}}=-\frac{1}{L} \frac{\partial^{2} \Omega_{\mathrm{av}}}{\partial x^{2}}= \begin{cases}\chi_{\mathrm{AF}}, & x=h_{s} \\ \kappa_{\mathrm{av}}, & x=\mu .\end{cases}
$$

The first derivative of $\Omega_{\mathrm{av}}$ with respect to $x$ yields,

$$
\frac{1}{L} \frac{\partial \Omega_{\mathrm{av}}}{\partial x}=-\frac{1}{2 \beta} \sum_{\alpha n} f_{\alpha}^{x} G_{\alpha n}
$$

where $f_{\alpha}^{\mu}=1$ and $f_{\alpha}^{h_{s}}=\alpha$. Differentiating once more, we obtain the desired susceptibilities (15). Note that these susceptibilities are evaluated at zero staggered field $h_{s}$, i.e., $h_{s}$ is put to zero once the second derivative of $\Omega_{\mathrm{av}}$ with respect to $\mu$ or $h_{s}$ is taken (in this case $\mu_{\alpha} \equiv \mu$ ). Thus, the susceptibilities are given by

$$
X_{\mathrm{av}}=\beta^{-2} \sum_{\alpha n, \alpha^{\prime} n^{\prime}} f_{\alpha}^{x} \Gamma_{n n^{\prime}, n^{\prime} n}^{\alpha \alpha^{\prime}} \gamma_{\alpha^{\prime} n^{\prime}}^{x}
$$

where $\Gamma$ is the local two-particle correlation function,

$$
\begin{aligned}
\Gamma_{n_{1} n_{1}^{\prime}, n_{2}^{\prime} n_{2}}^{\alpha \alpha^{\prime}}= & \int_{0}^{\beta} d \tau_{1} d \tau_{1}^{\prime} d \tau_{2} d \tau_{2}^{\prime} \exp \left\{i\left(\omega_{n_{1}} \tau_{1}+\omega_{n_{1}^{\prime}} \tau_{1}^{\prime}-\omega_{n_{2}^{\prime}} \tau_{2}^{\prime}-\omega_{n_{2}} \tau_{2}\right)\right\} \\
& \times\left[\left\langle\left\langle\psi_{\alpha}\left(\tau_{1}\right) \psi_{\alpha}^{*}\left(\tau_{2}\right) \psi_{\alpha^{\prime}}\left(\tau_{1}^{\prime}\right) \psi_{\alpha^{\prime}}^{*}\left(\tau_{2}^{\prime}\right)\right\rangle_{T}\right\rangle_{\mathrm{av}}-\left\langle\left\langle\psi_{\alpha}\left(\tau_{1}\right) \psi_{\alpha}^{*}\left(\tau_{2}\right)\right\rangle_{T}\left\langle\psi_{\alpha^{\prime}}\left(\tau_{1}^{\prime}\right) \psi_{\alpha^{\prime}}^{*}\left(\tau_{2}^{\prime}\right)\right\rangle_{T}\right\rangle_{\mathrm{av}}\right]
\end{aligned}
$$

The quantity $\gamma_{\alpha n}^{x}=\partial\left(G_{\alpha n}^{-1}+\Sigma_{\alpha n}\right) / \partial x$ in (16) measures the response of the averaged medium to an infinitesimal change of the field $x$. We will later see that $\gamma_{\alpha n}^{x}$ decides about the (in-)stability of a given phase. This dynamical response function is determined by an integral equation in frequency space, which does not explicitly depend on momentum. (Note that there are no convolutions in $\vec{k}$ space in the $d=\infty$ limit as is typical for a mean-field theory.) This property does not imply, however, that the response function $\gamma_{\alpha n}^{x}$ is local, too. It only indicates that $\gamma_{\alpha n}^{x}$ is diagonal in the momentum $\vec{q}$. A momentum dependence will enter by taking derivatives, e.g., of the local Green function, with respect to an external field with a particular $\vec{q}$ dependence $[\vec{q}=0$ in the case of the compressibility or the ferromagnetic susceptibility, and $\vec{q}=(\pi, \ldots, \pi)$ for the staggered susceptibility]. Infrared divergencies show up in the spectrum of $\gamma_{\alpha}^{x}(t)$.

The integral equations for $\gamma_{\alpha n}^{x}$ are derived as follows. Using the effective propagator $\mathcal{G}_{\alpha n}^{-1}=G_{\alpha n}^{-1}+\Sigma_{\alpha n}=(1+$ $\left.G_{\alpha n} \Sigma_{\alpha n}\right) / G_{\alpha n}$, such that

$$
\gamma_{\alpha n}^{x}=\partial \mathcal{G}_{\alpha n}^{-1} / \partial x
$$

we replace the self-energy in (13) by $\Sigma_{\alpha n}=\mathcal{G}_{\alpha n}^{-1}-G_{\alpha \sigma n}^{-1}$. Then (13) reads

$$
\begin{aligned}
& G_{\alpha n}=\left\langle g_{\alpha n}(E)\right\rangle_{E} \\
& G_{\alpha n}=-\int_{0}^{\beta} d \tau e^{i \omega_{n} \tau}\left\langle\left\langle\psi_{\alpha}(\tau) \psi_{\alpha}^{*}\left(\tau^{+}\right)\right\rangle_{T}\right\rangle_{\mathrm{av}}
\end{aligned}
$$

where $g_{\alpha n}(E)=\left(z_{\alpha n}-E^{2} / z_{-\alpha n}\right)^{-1}$, with $z_{\alpha n}=i \omega_{n}+$ $\mu+\alpha h_{s}-\mathcal{G}_{\alpha n}^{-1}+G_{\alpha n}^{-1}$, and

$$
\langle y(E)\rangle_{E}=\int_{\infty}^{\infty} d E N(E) y(E)
$$

To obtain $\gamma_{\alpha n}^{x}$ we differentiate (19) with respect to $x$. This yields

$$
\begin{aligned}
G_{\alpha n}^{\prime}= & \left(\gamma_{\alpha n}^{n}+\frac{G_{\alpha n}^{\prime}}{G_{\alpha n}^{2}}-f_{\alpha}^{x}\right) \xi_{\alpha n} \\
& +\left(\gamma_{-\alpha n}^{x}+\frac{G_{-\alpha n}^{\prime}}{G_{-\alpha n}^{2}}-f_{-\alpha}^{x}\right) \eta_{\alpha n}, \\
G_{\alpha n}^{\prime}= & \beta^{-1} \sum_{\alpha^{\prime} n^{\prime}} \Gamma_{n n^{\prime}, n^{\prime} n}^{\alpha \alpha^{\prime}} \gamma_{\alpha^{\prime} n^{\prime}}^{x},
\end{aligned}
$$

where $G_{\alpha n}^{\prime}=\partial G_{\alpha n} / \partial x$ and $\xi_{\alpha n}=\left\langle g_{\alpha n}^{2}(E)\right\rangle_{E}, \eta_{\alpha n}=$ $\left\langle E^{2} g_{\alpha n}^{2}(E)\right\rangle_{E} / z_{-\alpha n}^{2}$. Eliminating $G_{\alpha n}^{\prime}$ from (21), we obtain the following matrix equation for $\gamma_{+, n}^{x}$ and $\gamma_{-, n}^{x}$ :

$$
\beta^{-1} \sum_{\alpha^{\prime} n^{\prime}}\left(\beta \delta_{n n^{\prime}} R_{n^{\prime}}^{\alpha \alpha^{\prime}}+\Gamma_{n n^{\prime}, n^{\prime} n}^{\alpha \alpha^{\prime}}\right) \gamma_{\alpha^{\prime} n^{\prime}}^{x}=v_{\alpha n}^{x}
$$

The quantities $R_{n}^{\alpha \alpha^{\prime}}$ and $v_{\alpha n}^{x}$ are a matrix and a vector, respectively, with respect to the sublattice-spin index $\alpha$ and may be written as

$$
\begin{aligned}
\mathbf{R}_{n} & =-\left(\operatorname{det} \mathbf{D}_{n}\right)^{-1} \mathbf{D}_{n} \mathbf{T}_{n} \\
\vec{v}_{n}^{x} & =\mathbf{R}_{n} \cdot \vec{f}^{x}
\end{aligned}
$$

where

$$
\begin{gathered}
\mathbf{D}_{n}=\left(\begin{array}{cc}
1-\frac{\xi_{-, n}}{G_{-, n}^{2}} & \frac{\eta_{+, n}}{G_{-, n}^{2}} \\
\frac{\eta_{-, n}}{G_{+, n}^{2}} & 1-\frac{\xi_{+, n}}{G_{+, n}^{2}}
\end{array}\right) \\
\mathbf{T}_{n}=\left(\begin{array}{cc}
\xi_{+, n} & \eta_{+, n} \\
\eta_{-, n} & \xi_{-, n}
\end{array}\right), \\
\vec{f}^{x}=\left(\begin{array}{c}
1 \\
f_{-}^{x}
\end{array}\right) .
\end{gathered}
$$

In the paramagnetic phase, where $f_{\alpha}^{x} v_{\alpha n}^{x}=f_{-\alpha}^{x} v_{-\alpha n}^{x}$, Eqs. (23)-(25) reduce to (6) in Ref. 42. Equations (22)(24) determine the dynamical response function $\gamma_{\alpha n}^{x}$. The latter has a clear physical meaning. From (16) we see that the full susceptibility $(X)$ is essentially a product of a 
purely local, frequency-dependent part $(\Gamma)$ with a lattice dependent contribution $(\gamma)$, which therefore represents the surrounding of the single site under investigation.

To determine the boundary between the paramagnetic and the antiferromagnetic phase it is sufficient to calculate $\chi_{\mathrm{AF}}$ only in the paramagnetic phase. By contrast, $\kappa_{\mathrm{av}}$ has to be evaluated in both phases since in both phases a metal-insulator transition (MIT) is possible, in principle. Indeed, in the paramagnetic phase of a random alloy with discrete disorder spectrum, a MIT due to band-splitting may occur [at $U=0$ this is known to be the case, at least within CPA (Refs. 40 and 41)], and even in the presence of AFLRO, there are indications that both a metallic and an insulating phase exist. ${ }^{42}$ We will find that the most sensitive indicator for an incipient transition are the dynamic response functions $\gamma_{\alpha n}^{x}, x=\mu, h_{s}$ - especially their behavior close to the Fermi energy (i.e., their values for the lowest Matsubara frequencies), rather than $\chi_{\mathrm{AF}}$ and $\kappa_{\mathrm{av}}$ itself. The latter quantities are much less sensitive to changes in the ground state since they represent sums over the Matsubara frequencies.

\section{SPECIFICATION OF THE MODEL AND OF THE NUMERICAL PROCEDURE}

Equations (15)-(24) form the basis for the numerical evaluation of $\kappa_{\mathrm{av}}$ and $\chi_{\mathrm{AF}}$. Before they can be solved quantitatively, we have to specify the model parameters we use. The numerical calculations were performed with a semielliptic density of states (DOS), with total width $2 w$, i.e.,

$$
N(E)=\frac{2}{\pi w^{2}}\left(w^{2}-E^{2}\right)^{1 / 2} .
$$

This DOS is chosen because of its sharp algebraic band edges, resembling those typical for $d=3$, and its simple analytic form; it is exact for a Bethe lattice in the limit $Z \rightarrow \infty$.

To study the influence of the disorder we investigate, and compare, three qualitatively different distributions of random potentials.

(a) Discrete, binary-random-alloy distribution,

$$
P_{\text {binary }}\left(\epsilon_{i}\right)=\frac{1}{2} \delta\left(\epsilon_{i}-\frac{\Delta}{2}\right)+\frac{1}{2} \delta\left(\epsilon_{i}+\frac{\Delta}{2}\right) .
$$

The atomic potentials $\epsilon_{i}= \pm \Delta / 2$ are chosen with equal probability to ensure an average band filling of $n=1$ (only in this case, or $n \simeq 1$, is AFLRO expected to occur at all). This distribution is important since it leads to a disorder-induced MIT due to band splitting in the noninteracting system [an exact result in $d=\infty$ (Refs. 38 and 39)], which may compete with the interaction-induced Mott-Hubbard MIT.

(b) Continuous, semielliptic distribution,

$$
P_{\text {semi }}\left(\epsilon_{i}\right)=\frac{8}{\pi \Delta^{2}}\left[\left(\frac{\Delta}{2}\right)^{2}-\epsilon_{i}^{2}\right]^{1 / 2} .
$$

This is a much softer type of disorder. We found that a continuous, constant disorder distribution $P_{\text {const }}\left(\epsilon_{i}\right)=$ $\Delta^{-1} \theta\left(\frac{\Delta}{2}-\left|\epsilon_{i}\right|\right)$, leads to essentially identical results.

(c) Percolation-type disorder,

$$
P_{\text {perc }}\left(\epsilon_{i}\right)=(1-x) \delta\left(\epsilon_{i}\right)
$$

By using $P_{\text {perc }}\left(\epsilon_{i}\right)$ one may simulate doping in a nonrandom system with impurities, which do not hybridize with conduction electrons: with probability $x$ an infinite energy barrier is created, which prevents the electrons from visiting these sites. The three distributions allow us to test the universality of the magnetic behavior of the model obtained for different types of disorder.

To be able to study the competition between magnetic order caused by the electronic interactions and the disordering effects caused by the random potential, respectively, we work with an average band filling $n_{\mathrm{av}}=1$. Due to the symmetry of the distributions $(26 \mathrm{a}, \mathrm{b})$ in the random potentials $\epsilon_{i}$ under the replacement $\epsilon_{i} \rightarrow-\epsilon_{i}$, we can fix the chemical potential at $\mu=U / 2$. This can even be done for the distribution (26c): by considering only the accessible lattice sites, i.e., $L \rightarrow(1-x) L$ and $N_{\sigma} \rightarrow(1-x) N_{\sigma}$, where $N_{\sigma}$ is the number of electrons with spin $\sigma$, we are left again with a half-filled band because the Green function is itself weighted with an additional dilution factor of $1-x$.

For the numerical evaluation of the functional integral (14), we employ the algorithm of Hirsch and Fye. ${ }^{28}$ We discretize the time variable, i.e., $\beta=\Lambda \delta \tau$, with $0.25 \leq \delta \tau \leq 1$, and then extrapolate the quantities under investigation to $\delta \tau \rightarrow 0$. (Note that in the literature the number of time slices $\Lambda$ is often denoted by $L$ which we, however, use for the number of lattice sites.) To obtain a smooth imaginary-time Green function $G(\tau)$ even for discrete values of $\tau$, we use instead of $G_{n}$ [the Fourier transform of $G(\tau)$ with respect to Matsubara frequencies $\omega_{n}$ ] the function

$$
\begin{aligned}
\bar{G}_{n} & =\delta \tau /\left[1-\exp \left(\delta \tau / G_{n}\right)\right], \\
\bar{G}(\tau) & =\beta^{-1} \sum_{n} e^{i \omega_{n} \tau} \bar{G}_{n},
\end{aligned}
$$

with $\beta=\Lambda \delta \tau$. The same is done in the case of the inverse effective local propagator $\mathcal{G}_{n}^{-1}$, Eq. (8), leading to a smoothed quantity $\overline{\mathcal{G}^{-1}}(\tau)$. The latter is used in the Monte Carlo evaluation of the new Green function $\bar{G}^{\text {new }}(\tau)$ according to (13b). By inverting (27), we obtain the new function $G_{n}^{\text {new }}$, and from the new self-energy, $\Sigma_{n}^{\text {new }}=\mathcal{G}_{n}^{-1}-\left(G_{n}^{\text {new }}\right)^{-1}$, we obtain again the Green function $G_{n}$ by (13a). We then replace $G_{n}$ by $\bar{G}_{n}$ and continue the iteration procedure until self-consistency is reached.

In the continuum limit $\delta \tau \rightarrow 0$, the functions $\bar{G}_{n}$ and $G_{n}$ coincide. The definition (27) preserves the relation $G(\tau=0)=n-1$ even in the case of discrete $\delta \tau$ and suppresses unphysical and undesirable oscillations in $G(\tau)$, due to the discretization (see Fig. 1). In particular, by smoothing $G(\tau)$ in this simple way large-scale Fourier transformations (namely, for every iteration) described in Ref. 29 become unnecessary.

Exact summations over spin variables in the discrete 
Hubbard-Stratonovich transformation were used whenever possible, i.e., for $\Lambda \leq 22$. For $\Lambda>22$ we used Monte Carlo sampling. After typically 4-8 iterations an accuracy of $10^{-5}$ and $10^{-3}$ was reached in the exact summations and in the Monte Carlo sampling with $10^{4}$ sweeps per iteration, respectively. Close to the magnetic transition the convergence becomes significantly slower and the Monte Carlo sampling less and less efficient. This implies that in the immediate vicinity of the transition this method cannot be used to obtain accurate results.

The integration over the continuous disorder distributions was performed using the Gauss-Legendre quadrature. ${ }^{52} \mathrm{~A}$ discretization of the random energies in steps of $\delta \epsilon / w \sim 0.2$ gave an accuracy of $\sim 10^{-5}$ for all observables. The required CPU time increases proportional to $\Lambda^{2} 2^{\Lambda}$ and $\Lambda^{3}$ for the exact summation and the Monte Carlo sampling, respectively. ${ }^{53}$ Although the random energies break the particle-hole symmetry, we never encountered a minus-sign problem.

Setting $\hbar=k_{B}=1$, the only remaining physical dimension is that of an energy $(U, \Sigma, \Delta, T$, etc.) or inverse energy $\left(G, \kappa_{\mathrm{av}}, \chi_{\mathrm{AF}}, \beta, \delta \tau\right)$. Departing from our earlier convention, we now choose the half band width $w$ as our energy unit since it does not depend on the limiting process $Z \rightarrow \infty$. (This is in contrast to the scaling of the hopping amplitude $t=\alpha t^{*} / \sqrt{Z}$, where $\alpha$ may be cho-
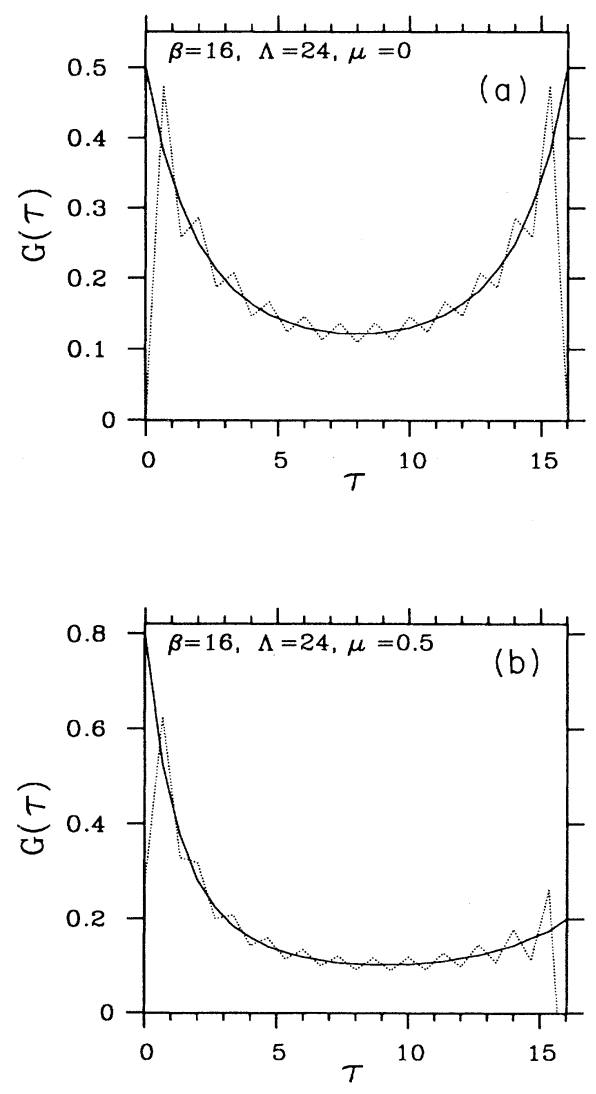

FIG. 1. Local Green function $G(\tau)$ for $U=0$ and $\mu=0$ (a), $\mu=0.5$ (b) obtained by usual Fourier transformation (dotted line) and by redefinition according to Eq. (13) (solid line). sen at will.) This convention agrees with that used by Kotliar and collaborators. ${ }^{21,23,30,32}$ To be able to compare the results presented in this paper with our earlier ones, ${ }^{42}$ all numerical values of quantities with dimension of energy (inverse energy) obtained in Ref. 42 must be divided (multiplied) by a factor of 2 . To compare with the results of Jarrell and co-workers ${ }^{24,25,33}$ and that of Georges and Krauth, ${ }^{29,31}$ their numbers have to be divided (multiplied) by a factor of $\sqrt{2}$.

\section{MAGNETIC PHASE DIAGRAM}

The competition between disorder and correlations in the Anderson-Hubbard model strongly affects its properties both in the ground state and at finite temperatures. Concerning the thermodynamics, the influence of different kinds of disorder on the stability of AFLRO near half filling is of particular interest. To determine the instability of the paramagnetic phase with respect to the formation of AFLRO we evaluate Eqs. (15)-(25) in the paramagnetic limit for $x=h_{s}$, in which case the dependence on the index $\alpha$ drops out; we set $\Sigma_{\alpha n} \equiv \Sigma_{n}$, etc., and $\gamma_{\alpha n}^{h_{s}} \equiv \gamma_{n}$. Note that the matrix equation (22) separates into two identical scalar equations in this case. As already mentioned below (17), it is not the averaged susceptibility itself, which is of primary interest in the investigation of the stability of the paramagnetic phase but the dynamic response function $\gamma_{n}$. The averaged susceptibility diverges (becomes negative), if and only if the real part of the response function $\gamma_{n}$ diverges for at least one frequency. This is characteristic for the dynamics of a quantum system of interacting particles possessing infinitely many coupled internal degrees of freedom labeled by the Matsubara frequencies. Each frequency corresponds to one mode in the quantum mechanical system which is described by complex variables. Note, however, that the Matsubara frequencies do not index the actual independent modes, since $\Gamma_{n n^{\prime}, n^{\prime} n}$ is not diagonal in frequency. Only the eigenvalues of the integral equation (22) represent the independent (eigen-)modes of the interacting quantum system. We can ascribe a critical (Néel) temperature $T_{N,|n|}$ to each eigenmode $n$ whereby $T_{N,|n|}>T_{N,\left|n^{\prime}\right|}$ if $|n|<\left|n^{\prime}\right|$. In Fig. 2, we show the function $\gamma_{n}(T)$, being a linear combination of eigenmodes for the lowest Matsubara frequencies, which lie very close to the diverging independent soft mode. The functions $\gamma_{n}(T)$ diverge due to the contribution of the eigenmode with the highest $T_{N,|n|}$. The highest critical temperature $T_{N,|n|}$ is the thermodynamic critical temperature $T_{N}$. Higher eigenmodes with lower critical temperature $T_{N,|n|}$ are physically unimportant for the static susceptibility $\chi_{\text {AF }}$ considered here. However, in the dynamical case some of the independent eigenmodes may mix, leading to a different dependence of $\gamma_{n}$ on $T$ in Fig. 2, e.g., the relation $T_{N,|n|}>T_{N,\left|n^{\prime}\right|}$ if $|n|<\left|n^{\prime}\right|$ may no longer hold.

Once the function $\gamma_{n}$ and the local vertex function $\Gamma_{n n^{\prime}, n^{\prime} n}$ are known, one may calculate the averaged susceptibility. Above $T_{N}$ this susceptiblity must obey the Curie-Weiss law, provided there is such a $T_{N}>0$. In 


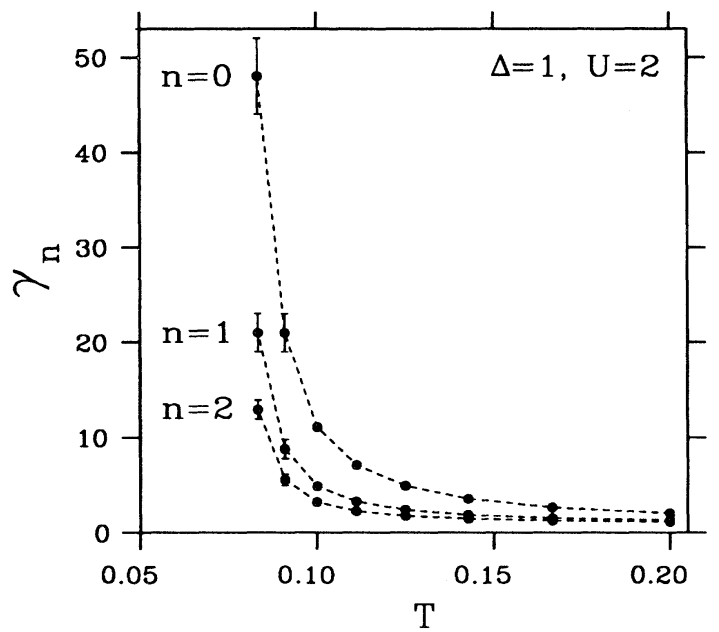

FIG. 2. Dynamical antiferromagnetic response function $\gamma_{n}$ vs temperature $T$. Binary alloy with disorder strength $\Delta=1$ and $U=2$. Here and in the following figures lines are usually guides for the eye, and error bars are roughly of the size of the symbols unless shown explicitly.

Fig. 3, three characteristically different temperature dependencies of $\chi_{\mathrm{AF}}^{-1}$ are shown for one value of (binary alloy) disorder, $\Delta=2$, at different interactions strengths. If $U / \Delta$ is sufficiently large the Curie-Weiss law with a finite $T_{N}$ is obeyed as in the case without disorder. For values of $U \sim \Delta$ the low-temperature behavior begins to be determined by the scattering off the frozen random configurations and deviations from the Curie-Weiss-law become apparent. For $U / \Delta<1$, we observe a minimum in $\chi_{\mathrm{AF}}^{-1}$ (i.e., a maximum in $\chi_{\mathrm{AF}}$ itself), which separates the temperature-dominated regime from the disorder-dominated regime. In the latter case the longrange correlations are continually suppressed, due to impurity scattering. The phase diagram in the $T-U$ plane calculated from the zeros of $\chi_{\mathrm{AF}}^{-1}$ is plotted in Fig. 4(a) for different values of the binary-alloy disorder. We can distinguish two different regimes. (a) $U<U_{c} \simeq 2.5$ : the disorder gradually suppressed the long-range order and

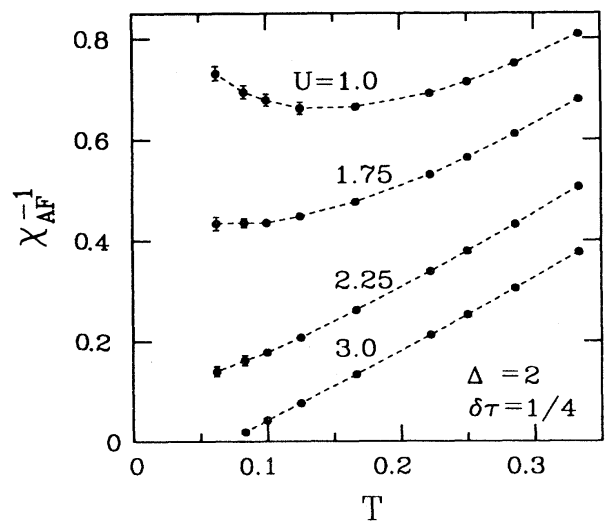

FIG. 3. Inverse averaged antiferromagnetic susceptibility $\chi_{\mathrm{AF}}^{-1}$ vs $T$ for the binary alloy with $\Delta=2$ and several values of $U$.
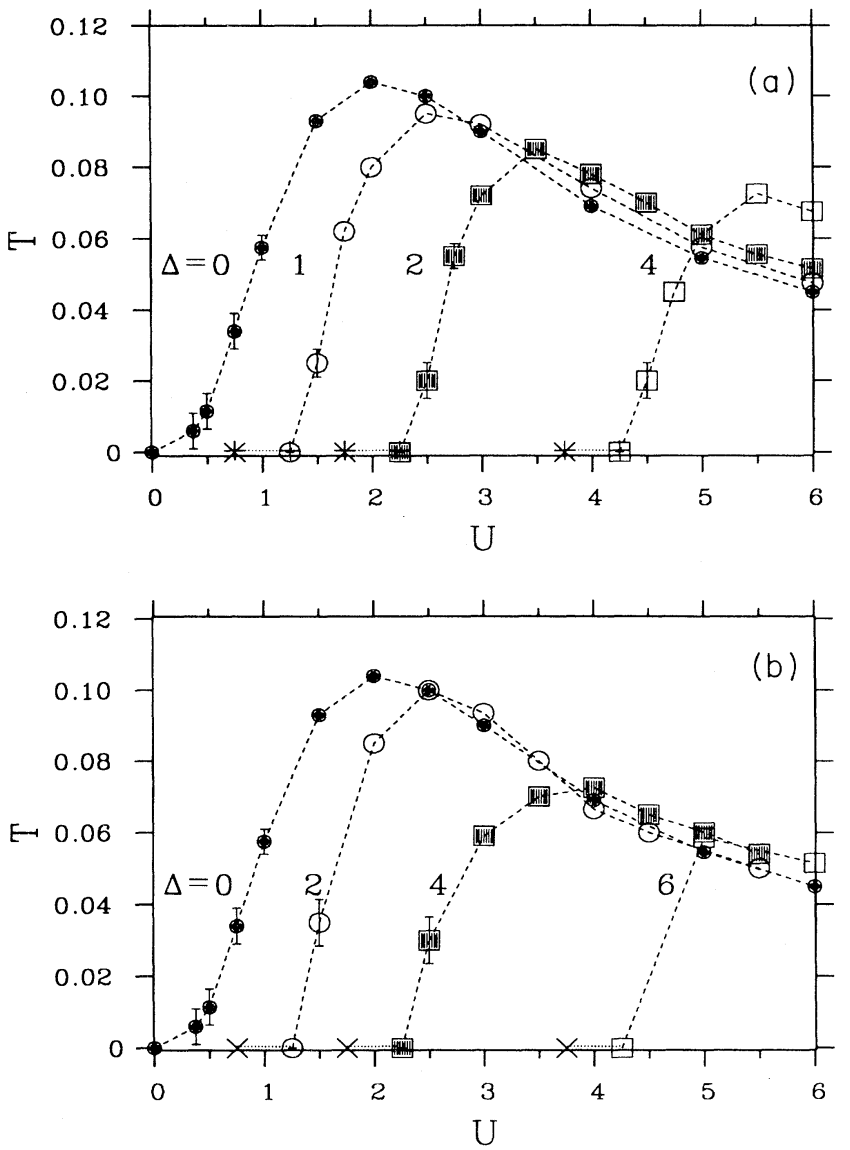

FIG. 4. (a) $T-U$-phase diagram for the binary alloy with $\Delta=0,1,2,4$ obtained from the zeroes of $\chi_{\mathrm{AF}}^{-1}$ (see Fig. 3). The AF phase is stable below the curves. The dotted lines at $T=0$ depict the regimes where the Curie law would give negative transition temperatures. Below the crosses $\chi_{\mathrm{AF}}^{-1}$ has no zeroes but a minimum and an $\mathrm{AF}$ phase can no longer be expected. (b) $T-U$-phase diagram for the semielliptic distribution of the random energies with width $\Delta=0,2,4,6$.

thus reduces the critical temperature. This is exactly what one expects from scattering off frozen random configurations; (b) $U>U_{c}$ : here, the situation is strikingly different since $T_{N}$ curves of constant disorder start to cross. This means that a small amount of disorder supports the formation of AFLRO, i.e., the critical temperature increases with disorder as shown in Fig. 5. A maximal critical temperature is reached at some value $\Delta_{c}(U)$ beyond which a further increase of disorder causes the critical temperature to decrease monotonically to zero. This effect is particularly pronounced at strong coupling. It is also observed, but less pronounced, in the case of the continuous disorder distribution [Fig. 4(b)]. In the limit $U \gg \Delta, t$ the enhancement of $T_{N}$ may be explained as follows. ${ }^{54}$ The virtual hopping of an electron with spin $\sigma$ from a given site $A$ with local energy $\epsilon_{A}$ to a neighboring site $B$ with energy $\epsilon_{B}$ occupied by a $(-\sigma)$ electron leads to an energy gain $J_{1}=-t^{2} /\left[U-\left(\epsilon_{A}-\epsilon_{B}\right)\right]$ and to $J_{2}=-t^{2} /\left[U+\left(\epsilon_{A}-\epsilon_{B}\right)\right]$ for the reverse process. The effective spin coupling $J\left(\epsilon_{A}, \epsilon_{B}\right)$ is given by the sum of 


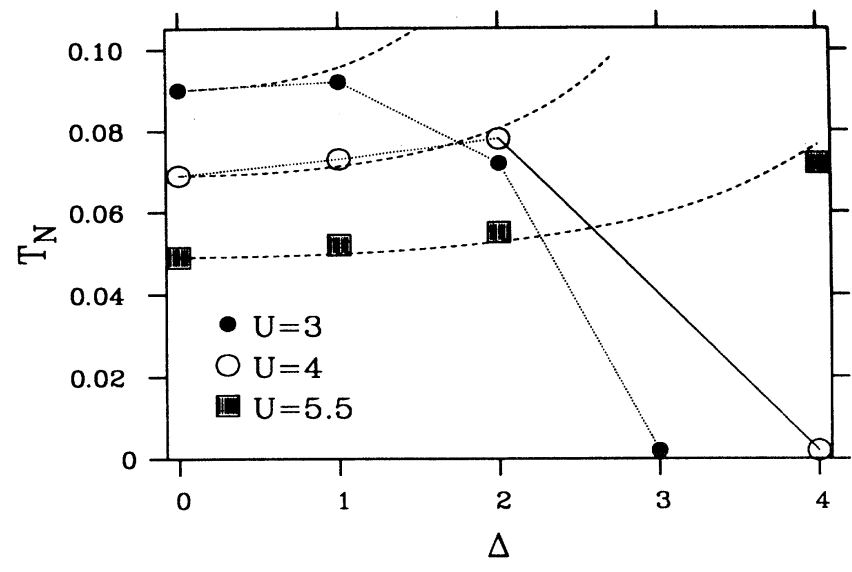

FIG. 5. $T$ vs $\Delta$ for the binary-alloy distribution at $U=3,4$, and 5.5. Dashed lines: quadratic increase of $T_{N}$ according to Eq. (29b) with $\lambda=1 / 2$; dotted lines are guides to the eye only.

these energies, $J\left(\epsilon_{A}, \epsilon_{B}\right)=J_{1}+J_{2}$, which for a bounded disorder distribution, $-\frac{\Delta}{2} \leq \epsilon_{A, B} \leq \frac{\Delta}{2}$, with $U \gg \Delta$ (strong coupling) implies

$$
J\left(\epsilon_{A}, \epsilon_{B}\right)=\frac{2 t^{2}}{U}\left[1+\left(\frac{\epsilon_{A}-\epsilon_{B}}{U}\right)^{2}\right] .
$$

Assuming that even in the presence of disorder the Néel temperature $T_{N}(U, \Delta) \propto<J\left(\epsilon_{A}, \epsilon_{B}\right)>_{\text {av }}$, we find

$$
\begin{aligned}
\frac{T_{N}(U, \Delta)}{T_{N}(U, 0)} & =\int d \epsilon_{A} \int d \epsilon_{B} J\left(\epsilon_{A}, \epsilon_{B}\right) P\left(\epsilon_{A}\right) P\left(\epsilon_{B}\right) \\
& =1+\frac{2}{U^{2}}\left[p_{2}-p_{1}^{2}\right]
\end{aligned}
$$

for arbitrary disorder distribution $P(\epsilon) . p_{1}$ and $p_{2}$ are the first and second moment of $P(\epsilon)$, where $p_{l}=\int d \epsilon \epsilon^{l} P(\epsilon)$. For a symmetric, bounded distribution $\left(p_{1}=0\right)$ one, therefore, finds

$$
\frac{T_{N}(U, \Delta)}{T_{N}(U, 0)}=1+\lambda\left(\frac{\Delta}{U}\right)^{2},
$$

with $\lambda=2 p_{2} / \Delta^{2}$, i.e., the disorder is indeed found to $i n$ crease $T_{N}$ irrespective of the type of disorder. This effect is the more pronounced the more structure $P(\epsilon)$ has at $|\epsilon| \lesssim \frac{\Delta}{2}$, i.e., is larger for the binary alloy $\left(\lambda=\frac{1}{2}\right)$ than for the semielliptic $\left(\lambda=\frac{1}{8}\right)$ distribution. This quadratic increase of $T_{N}$ with $\Delta$ is indeed found numerically at large $U$ (Fig. 5). Apparently, the mechanism that enhances $T_{N}$ is effective already at $U$ values as small as $U \sim 2.5$. It is interesting to note that for given strength of disorder $\Delta$ the $U$ value, where AFLRO begins to set in, $U_{\text {min }}$, given by $T_{N}\left(U_{\min }, \Delta\right)=0$, tracks almost perfectly with $\Delta$, i.e., $U_{\min }(\Delta) \simeq \Delta$. More generally, for binary-alloy disorder the $T_{N}$ curves are found to obey the phenomenological scaling law,

$$
\begin{aligned}
T_{N}(U, \Delta) \simeq & T_{N}\left(U\left[1-\left(\frac{\Delta}{U}\right)^{2}\right], 0\right) \\
& \times\left[1-\frac{1}{2}\left(\frac{\Delta}{U}\right)^{2}\right]
\end{aligned}
$$

for arbitrary $U$ (Fig. 6). For $U \gg \Delta,(31)$ reduces to (29b).

It is well known that AFLRO may be destroyed by doping the (nonrandom) system with holes, such that $n_{\text {av }}<1$. This is a very interesting effect both from an experimental and theoretical point of view. The effect of adding holes may be approximated by introducing percolation-type disorder, (26c), where the random siteenergy is zero with probability $1-x$ and infinite with probability $x$. Since in $d=\infty$ the critical value for percolation is $x=1$ (Ref. 55), AFLRO will persist up to $x=1$. The boundaries between the paramagnetic and the antiferromagnetic phases for this type of disorder are shown in Fig. 7. At weak coupling, the critical temperature remains constant over a wide range of $x$ and then rapidly falls to zero. At strong coupling, however, the critical temperature monotonically decreases and approaches the linear behavior $T_{N}(x)=T_{N}(0)(1-x)$. The latter dependence of $T_{N}$, which is very different from the one observed in the previous two cases, is an exact result in the case of the disordered Falicov-Kimball model. ${ }^{39}$

The magnetic correlations are mediated by the local magnetic moments. Their static average, $m_{\mathrm{av}}$, is defined by $m_{\mathrm{av}}^{2} \equiv L^{-1} \sum_{i}\left\langle\left\langle\left(\hat{n}_{i \uparrow}-\hat{n}_{i \downarrow}\right)^{2}\right\rangle_{T}\right\rangle_{\mathrm{av}}=1-2 d_{\mathrm{av}}$. Here, $d_{\mathrm{av}}=L^{-1} d \Omega_{\mathrm{av}} / d U$ is the average double occupancy of lattice sites. For the binary and the semielliptic disorder distributions discussed above, $m_{\text {av }}^{2}$ is shown as a function of $U$ in Fig. 8(a). The disorder is seen to have two main effects which are independent of the specific disorder distribution: (1) at a fixed value of $U$, an increase of the disorder always reduces the moments. (2) For fixed disorder strength $\Delta$, an increase of $U$ always enhances the moments, with saturation starting at $U \gtrsim \Delta$. Both features are easily explained in terms of the effect the disorder and the on-site repulsion, respectively, have on the average double occupancy $d_{\text {av }}$. For $U \ll \Delta$,

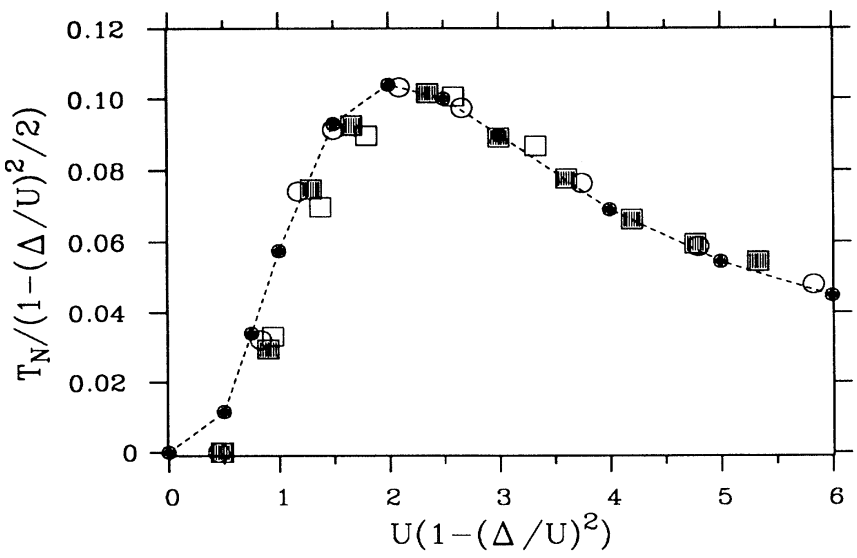

FIG. 6. Scaling plot of $T_{N}$ for the binary-alloy distribution according to Eq. (30); symbols as in Fig. 4(a). 


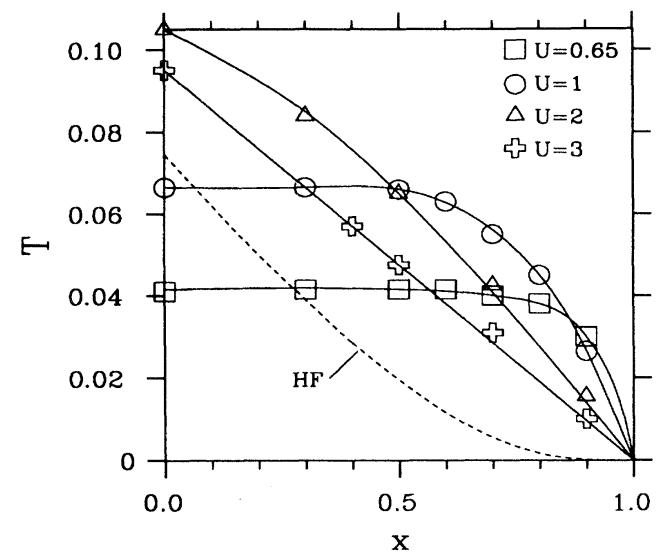

FIG. 7. $T-x$-phase diagram for the percolation-type disorder for several values of $U$. The dashed line depicts the Hartree-Fock (HF) result for the smallest value of $U=0.65$.
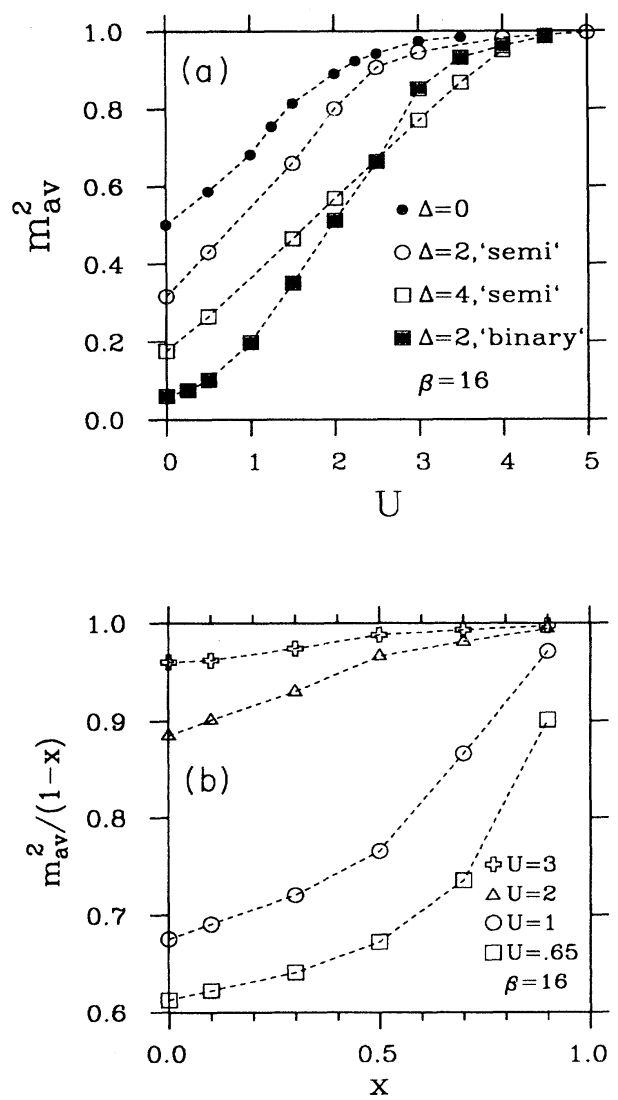

FIG. 8. (a) Averaged quadratic local moment $m_{\mathrm{av}}^{2}$ vs $U$ at inverse temperature $\beta=16$. Without disorder (full circle); binary alloy with $\Delta=2$ (full square); semielliptic distribution with $\Delta=2$ (open circle) and $\Delta=4$ (open square). (b) Averaged quadratic local moment $m_{\mathrm{av}}^{2} /(1-x)$ (normalized to the concentration of sites with random energy zero) vs $x$ for the percolation-type disorder at $\beta=16$ for several values of $U$. the local repulsion is weak, while the spatial fluctuations of the atomic potentials are strong, such that $d_{\text {av }}$ is at its maximum value $\left(d_{\mathrm{av}} \simeq 1 / 2\right)$ and $m_{\mathrm{av}}$ is small. As $U$ increases the particles are forced to separate and $d_{\mathrm{av}}$ decreases, i.e., $m_{\mathrm{av}}$ increases, too. The local magnetic moments do not show a critical behavior close to the transition temperature $T_{N}$. In the case of the percolation-type disorder [Fig. 8(b)], the density of local moments on the reduced lattice with $(1-x) L$ sites increases with concentration $x$. In other words, in the presence of disorder, the saturation of $m_{\text {av }}^{2}$ sets in at smaller values of $U$ than in the pure system. The influence of the percolation-type disorder can be explained by the reduction of the kinetic energy by a factor of $1-x$, which is due to the reduced average number of nearest neighbors. For large $U$, in the Heisenberg limit, the factor $t^{2}$ in the antiferromagnetic coupling, $J \sim t^{2} / U$, has to be replaced by $t^{2}(1-x)$ leading to a linear decrease of $T_{N}$. For small values of $U$, the influence of the repulsion increases due to the reduction of the kinetic energy. Therefore, the double occupancy is suppressed, i.e., $m_{\mathrm{av}}^{2}$ is enhanced by the percolation disorder. The enhancement of $m_{\mathrm{av}}^{2}$ leads to the stabilization of AFLRO. This effect is definitely not described within the Hartree-Fock approximation; the latter only leads to an exponential suppression of $T_{N}$ if $U$ or $(1-x)$ becomes small.

In Fig. 9, the temperature dependence of the order parameter (the averaged staggered magnetization $M_{\mathrm{AF}}$ ) is shown. In the vicinity of the transition point, $T \lesssim T_{N}$, it is well represented by a mean-field-type dependence $M_{\mathrm{AF}}(T) \propto\left(T_{N}-T\right)^{1 / 2}$ indicated by the dashed line. We observe that the extrapolation of the Monte Carlo data to $M_{\mathrm{AF}}\left(T_{N}\right)=0$, using this law leads to the same critical temperature as that obtained from the divergence of $\chi_{\mathrm{AF}}$, i.e., $\chi_{\mathrm{AF}}^{-1}\left(T_{N}\right)=0$, assuming a Curie-Weiss law for $T \gtrsim T_{N}$. Deviations from the square-root behavior of $M_{\mathrm{AF}}(T)$ set in at lower temperatures.

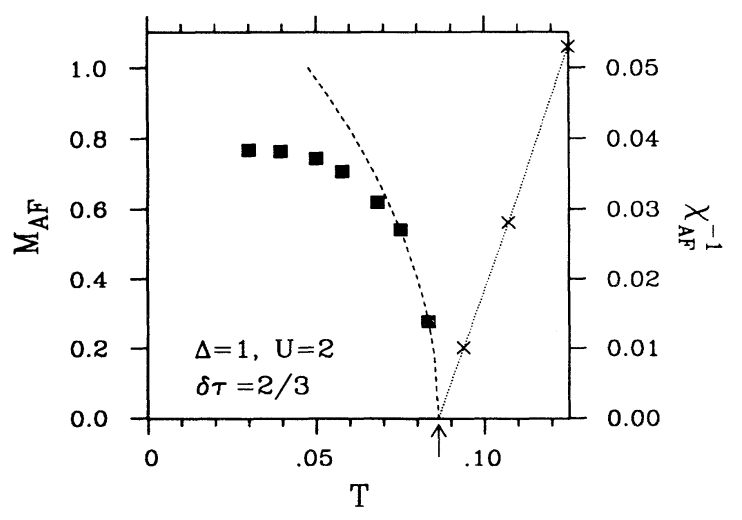

FIG. 9. Staggered magnetization $M_{\mathrm{AF}}$ and inverse averaged susceptibility $\chi_{\mathrm{AF}}^{-1}$ vs $T$ for the binary alloy with $\Delta=1$, $U=2, \delta \tau=2 / 3$. Dashed line: square-root fit of the last two points of $M_{\mathrm{AF}}$ below the transition; dotted line: linear fit of $\chi_{\mathrm{AF}}^{-1}$. The arrow indicates the extrapolated Néel temperature. 


\section{METAL-INSULATOR TRANSITIONS}

In the preceding section, we found that at weak coupling the disorder suppresses the AFLRO. In the ground state, the system is then a disordered paramagnet. Whether it is metallic or not depends on the type and strength of the disorder. In $d=\infty$, where Anderson localization does not occur, ${ }^{38,39}$ a paramagnetic insulator only forms if the spectrum of the disorder distribution is multiple connected, such as in the case of the binaryalloy disorder. For this type of randomness, a MIT due to band splitting is expected to occur at some value $\Delta \geq 1$. In the noninteracting case, $U=0$, the exact result in $d=\infty$ [obtained by CPA (Ref. 40)] is $\Delta=1$.

For $U=0$, there are two equivalent criteria to decide on whether the ground state in $d=\infty$ is metallic or insulating. They correspond to (i) a spectral definition of an insulator, based on the disappearance of the DOS at the Fermi level (assuming that only extended electrons are present), and (ii) a thermodynamic definition, employing the disappearance of the compressibility ${ }^{56}$ However, in interacting systems these two criteria need not coincide. Hence, we will investigate both. In Fig. 10(a), the averaged compressibility $\kappa_{\text {av }}$ of interacting electrons in the presence of binary-alloy disorder is shown as a function of $U$ for $\beta=16$. Hence, $\kappa_{\mathrm{av}}$ is calculated across the line $T=0.0625$ in Fig. 4(a), i.e., within the paramagnetic and the antiferromagnetic phase, respectively (the transition points are indicated by arrows). For a given $\Delta \gtrsim 1$, the curves display a common behavior as a function of $U: \kappa_{\text {av }}$ is (exponentially) small at $U=0$, then increases and, at $U \simeq \Delta$, approaches a maximum, beyond which it becomes (exponentially) small again for $U \gg \Delta$. This behavior has a clear physical interpretation: For $U \ll \Delta$, with $\Delta \gtrsim 1$, the double occupancy of lattice sites is at its maximum value $\left(d_{\mathrm{av}} \simeq \frac{1}{2}\right)$, at the same time the $\mathrm{ki}$ netic energy cannot delocalize these states. Hence, the system is insulating. At $T=0$ and $U=0$, this is an exact property for $\Delta>1$ (split-band limit) in $d=\infty$. As $U$ increases, the on-site Coulomb repulsion forces the particles to separate from each other. As a consequence, the particles become less localized. We then expect that, at some critical value $U_{c}^{M I, 1}$, a macroscopic fraction of the electrons becomes extended and the system starts to be metallic. This real-space picture has its analogy in $\vec{k}$ space (or with respect to energy): as the interaction increases the previously separated bands change their shapes, i.e., the interaction-induced energy exchange between particles leads to a transfer of states into the energy gap, where the Fermi energy is located. At the critical value $U_{c}^{M I, 1}$ the (still algebraic) band edges reach the Fermi level, producing a finite DOS there as well as a finite overall compressibility. (We cannot rule out that $U_{c}^{M I, 1}=0$, i.e., that the DOS at the Fermi level is finite even at arbitrarily small $U$; however, this would require the bands to acquire exponential tails - a feature which cannot be observed numerically.) Hence, in contrast to an interacting system without disorder, the Coulomb interaction in a disordered system is able to improve the metallicity of the system and may even turn an insulator into a metal. Apparently, the interaction-induced energy exchange smooths the energy spectrum of the disordered system, thus leading to an easier transfer of energy.

For $U>U_{c}^{M I, 1}$ the system is then expected to be a metal without Fermi liquid properties [since $\operatorname{Im} \Sigma(\omega=$ $0) \neq 0$, i.e., the quasiparticles have a finite lifetime at the Fermi level], at least in $d=\infty$. As $U$ is further increased the DOS and $\kappa_{\mathrm{av}}$ increase, reaching a maximum at $U \simeq \Delta$. For even larger $U$, the effect of pushing states into the gap is reversed and $\kappa_{\mathrm{av}}$ decreases again. Hence, at some critical value $U_{c}^{M I, 2}$ a second transition occurs, back into an insulating state, where doubly occupied sites are almost completely suppressed, whereby the mobility is obstructed by the repulsive interaction.

In Fig. 10(b), $\kappa_{\text {av }}$ for the continuous, semielliptic disorder distribution is shown. Here, $\kappa_{\mathrm{av}}$ behaves qualitatively as in the nonrandom case, i.e., $\kappa_{\mathrm{av}}$ decreases monotonically since the DOS does not split at $U=0, T=0$. This reduction of $\kappa_{\mathrm{av}}$ occurs in two steps: for $U \lesssim \Delta$ $\kappa_{\text {av }}$ decreases almost linearly with $U$, corresponding to
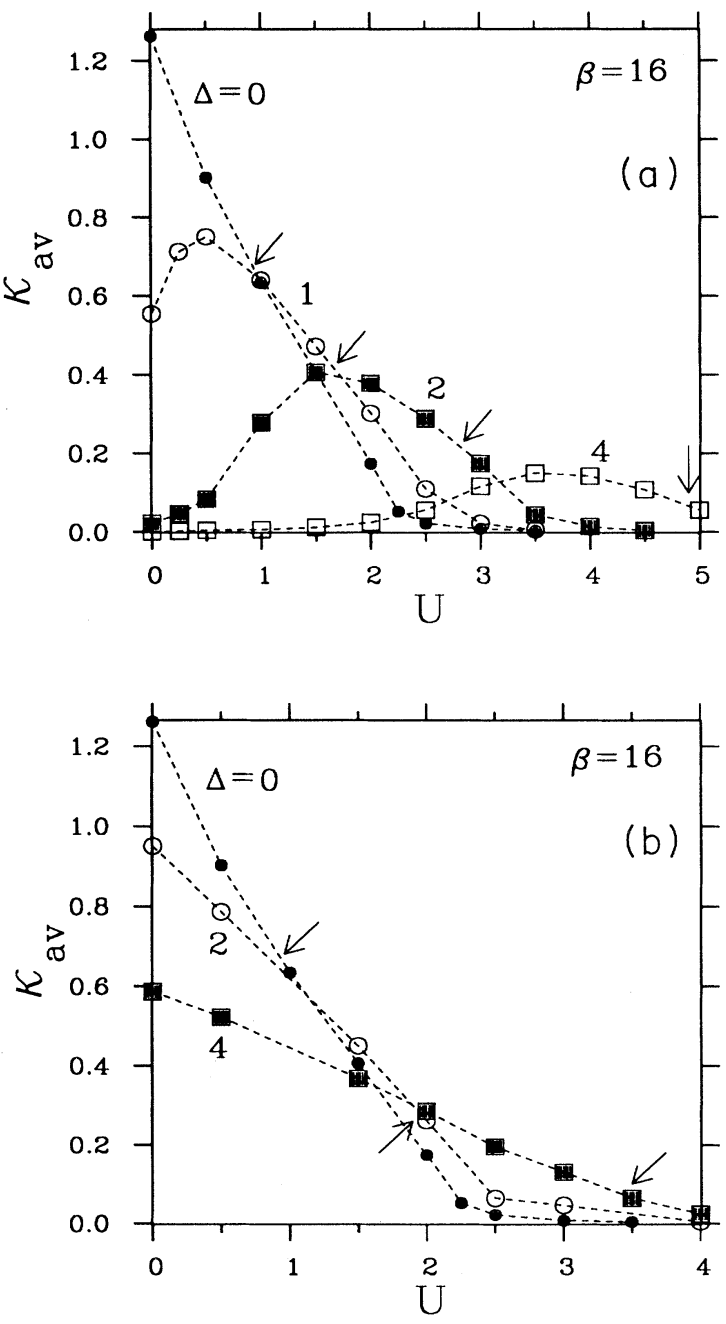

FIG. 10. Averaged compressibility $\kappa_{\mathrm{av}}$ vs $U$ at $\beta=16$. (a) Binary alloy, $\Delta=0,1,2,4$; (b) semielliptic distribution with $\Delta=0,2$, and 4 . Arrows indicate the transition to the antiferromagnetic state. 
the linear suppression of doubly occupied sites $d_{\mathrm{av}}$, or $m_{\text {av }}^{2}$ [see Fig. 8(a)]; in this region the system is metallic but not a Fermi liquid. Then, for $U \gtrsim \Delta$, when the number of doubly occupied sites is almost zero so that the local moments are almost saturated, $\kappa_{\mathrm{av}}$ approaches zero (exponentially) slowly. Strictly speaking, at finite temperatures, one has $\kappa_{\mathrm{av}}>0$ for $U<\infty$.

Next, we discuss the relation of $U_{c}^{M I, 1}$ and $U_{c}^{M I, 2}$ to the critical interaction strength $U_{c}^{\mathrm{AF}}$, where the paramagnet becomes unstable with respect to AFLRO. In Fig. 10(a),(b), the position of $U_{c}^{\mathrm{AF}}$ is indicated by an arrow. Since the local moments vanish in the (binaryalloy)-disorder-induced insulating phase, the insulatorto-metal transition at $U_{c}^{M I, 1}$ occurs before the magnetic order sets in, i.e., $U_{c}^{M I, 1}<U_{c}^{\mathrm{AF}}$. This raises the question of what happens as one goes through the magnetic transition into the ordered phase: will the compressibility jump to zero discontinuously or will there be an antiferromagnetic metal, i.e., does the interaction-induced MIT coincide with the magnetic transition or not? To answer this question the behavior of $\kappa_{\mathrm{av}}$ near the transition point has to be investigated. In Fig. 11(a), the change of $\kappa_{\mathrm{av}}$ due to the onset of AFLRO in the case of binary-alloy disorder is shown. The results for the paramagnetic phase are com-
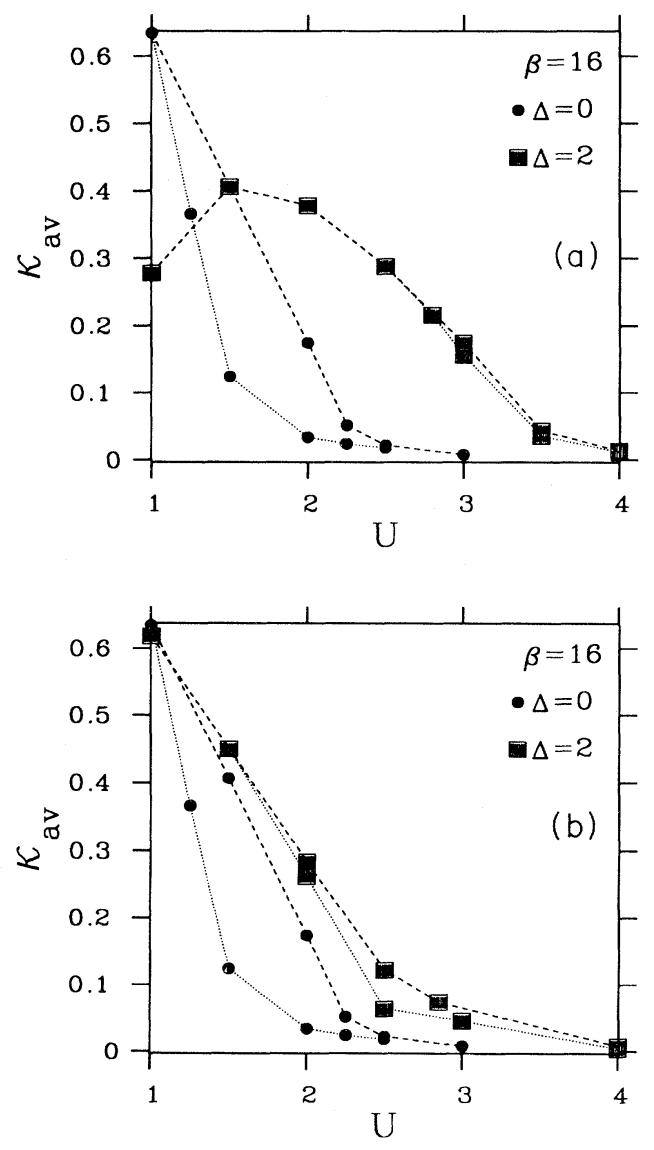

FIG. 11. Averaged compressibility $\kappa_{\text {av }}$ vs $U$ at $\beta=16$ in the paramagnetic phase (dashed lines) and in the antiferromagnetic phase (dotted lines). (a) Binary alloy, (b) semielliptic distribution. pared with those for the ordered phase. At $\Delta=0$, the AFLRO is seen to suppress $\kappa_{\text {av }}$ drastically. By contrast, at finite disorder $(\Delta=2)$, this difference almost vanishes. Apparently the disorder stabilizes the metallic state close to $U_{c}^{\mathrm{AF}}$. A similar behavior is observed for the continuous disorder [Fig. 11(b)]. These results suggest that in the vicinity of $U_{c}^{\mathrm{AF}}$ the system is an antiferromagnetic metal. However, MC techniques are not able to decide whether this is true even at $T=0$. We attempted to extrapolate our finite-temperature data to $T=0$, but could not find a simple, accurate extrapolation law. In Fig. 12, the averaged compressibility for the system with binary-alloy disorder, calculated at $T=1 / 16$ and $1 / 40$, is shown. Although at the lower temperature $\kappa_{\text {av }}$ is lower and the slope is slightly steeper at the transition point, the critical point (indicated by an arrow) seems to be well inside the metallic phase. In particular, the tail behavior for $U>U_{c}^{\mathrm{AF}}$ does not show any significant change.

To investigate whether, and how, an insulator evolves, it is instructive to plot the dynamical response function $\gamma_{\alpha n}$, or the quantities

$$
\kappa_{n}:=\frac{1}{2} \sum_{\alpha} \partial G_{\alpha n} / \partial \mu,
$$

with

$$
\kappa_{\mathrm{av}}=\beta^{-1} \sum_{n} \kappa_{n}
$$

as a function of Matsubara frequency $\omega_{n}=\pi T(2 n+1)$. In Fig. 13(a), the real part of $\kappa_{n}$ is shown for a system without disorder, $\Delta=0$, for $U=1.75$ and $T=1 / 64$ [the system is then well inside the antiferromagnetic region, see Fig. 4(a)]. The behavior of $\operatorname{Re} \kappa_{n}$ close to $\omega_{n}=0$ is seen to be very different for the antiferromagnetic and the (hypothetical) paramagnetic solution. For the paramagnetic solution, which is metallic, $\operatorname{Re} \kappa_{n}$ is a monotonically decreasing function of $\left|\omega_{n}\right|$. By contrast, in the antiferromagnetic (i.e., insulating) phase, this is only so for $\left|\omega_{n}\right| \gtrsim 1$, while for $\omega_{n}$ close to zero, $\operatorname{Re} \kappa_{n}$ becomes

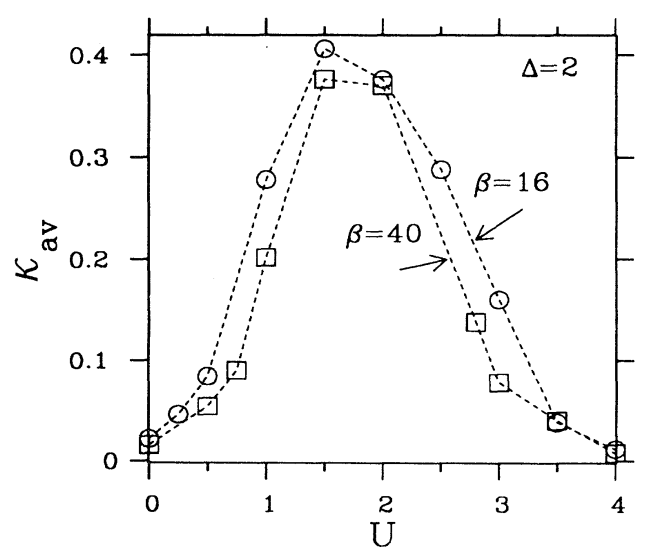

FIG. 12. Averaged compressibility $\kappa_{\text {av }}$ vs $U$ for the binary alloy at $\beta=16$ and 40 . Arrows indicate the transition to the antiferromagnetic state. 
strongly negative. In the former case, the sum over $\kappa_{n}$, i.e., $\kappa_{\mathrm{av}}$, is then clearly positive, while in the latter case $\kappa_{\text {av }}$ becomes very small due to the negative contributions close to $\omega_{n}=0$. This behavior at $\Delta=0$ is now contrasted with that in the presence of binary-alloy disorder $[\Delta=2$, see Fig. $13(\mathrm{~b})]$ at $U=2.65$ for two different temperatures: $T=1 / 20$ [close to the magnetic transition] and $T=1 / 64$ [well inside the ordered phase; see Fig. 4(a)]. At $T=1 / 64$, the range of $\omega_{n}$ values for which $\operatorname{Re} \kappa_{n}<0$ is now even narrower than in the case without disorder. This shows that a definite answer to the question of whether the antiferromagnetic phase close to $U_{c}^{\mathrm{AF}}$ is insulating $\left(\kappa_{\mathrm{av}}=0\right)$ or not at $T=0$ can only be obtained from the behavior of $\operatorname{Re} \kappa_{n}$ at very small $\omega_{n}$, i.e., from $\kappa_{\mathrm{av}}$ at very low temperatures $\left(T<10^{-2}\right)$. Within the MC approach used here, such low temperatures are not attainable.

The situation is similar in the case of $-\operatorname{Im} G_{n}$, the imaginary part of the one-particle Green function $G_{n}=$ $\frac{1}{2} \sum_{\alpha} G_{\alpha n}$, which yields a spectral condition for an insulator (for $n=0$ and in the limit $T \rightarrow 0$, the function $-\operatorname{Im} G_{0} \pi$ coincides with the DOS at the Fermi level). If $\operatorname{Im} G_{0} \rightarrow 0$ for $T \rightarrow 0$ the ground state of the system is insulating. In Fig. 14, the influence of the onset of AFLRO
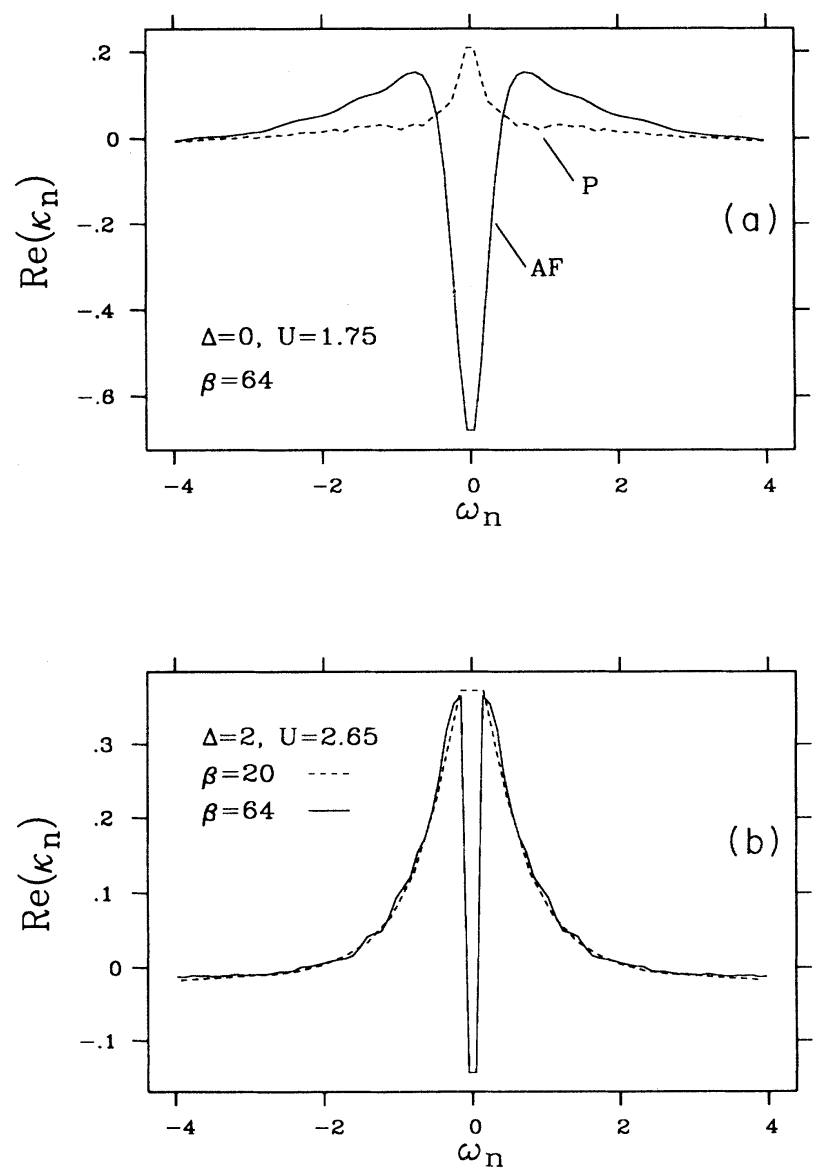

FIG. 13. Real part of $\kappa_{n}$ vs $\omega_{n}$, (a) for $\Delta=0, U=1.75$ and $\beta=64$ in the paramagnetic phase $(P)$ and the antiferromagnetic phase (AF); (b) for the binary alloy with $\Delta=2$, $U=2.65$ in the AF phase at $\beta=20$ and 64 .

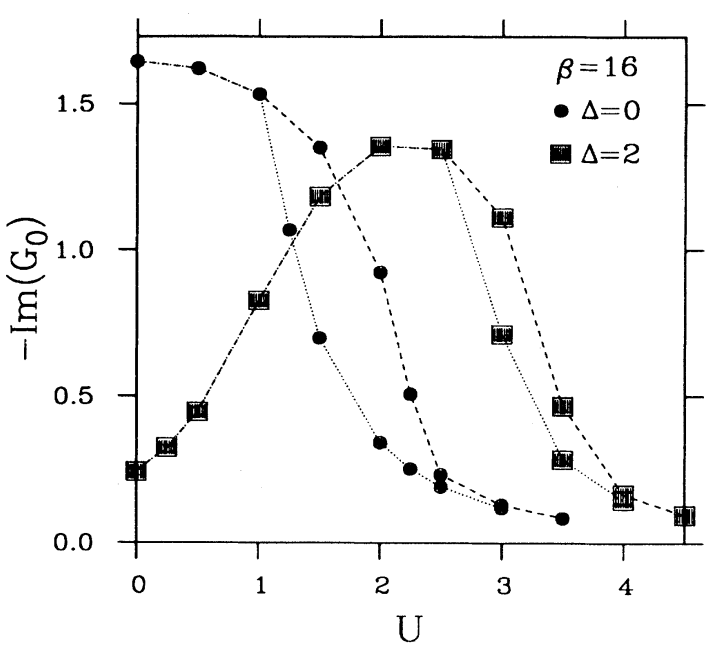

FIG. 14. Imaginary part of the one-particle Green function $G_{n}$ at the lowest Matsubara frequency $\omega_{0}=\pi T$ vs $U$ for a system without disorder $(\Delta=0)$ and with binary-alloy disorder $(\Delta=2)$ at $\beta=16 ; P$ phase (dashed lines), AF phase (dotted lines).

on $-\operatorname{Im} G_{0}$ is shown. Although in the disordered system the difference between the magnetic and nonmagnetic solution is now slightly stronger than in the case of $\kappa_{\text {av }}$ [see Fig. 11(a)], the magnetic transition occurs where $\operatorname{Im} G_{0}$ is large, i.e., well inside the metallic phase. In Fig. 15, the behavior of $-\operatorname{Im} G_{n}$ at the few lowest values of $\omega_{n} \geq 0$ is plotted for the same values of $U$ and $\beta$ as for $\operatorname{Re} \kappa_{n}$ in Fig. 13. In the case without disorder [Fig. 15(a)], this behavior is qualitatively similar to that of $\operatorname{Re} \kappa_{n}$ [Fig. 13(a)]: the paramagnetic solution is clearly metallic, while the antiferromagnetic phase is insulating since $\operatorname{Im} G_{0} \rightarrow 0$. In the presence of disorder [Fig. 15(b)], however, the downturn of $-\operatorname{Im} G_{n}$ in the phase with AFLRO $(\beta=64)$ sets in only very close to $\left|\omega_{n}\right| \simeq 0$, i.e., at very low temperatures. If close to the transition, the antiferromagnetic phase were really insulating the decrease of $-\operatorname{Im} G_{n}$ will have to be very rapid. In Fig. 16, we plotted our Monte Carlo data for $-\operatorname{Im} G_{0}$ in the case of binary-alloy disorder with $\Delta=2$ as a function of temperature for several values of $U$. Both at very small $U$ (i.e., $U<1$, where the disorder-induced gap of the DOS dominates the behavior) and at large $U$ (Heisenberg-limit) the temperature dependence may be clearly extrapolated to $-\operatorname{Im} G_{0}=0$ for $T=0$, i.e., the ground state is insulating. However, for $U \simeq U_{c}^{\mathrm{AF}}(\Delta, T=0)$, with $U_{c}^{\mathrm{AF}}(2,0) \simeq 2-2.5$, these results cannot be safely extrapolated. Hence, the question whether in the disordered system close to the magnetic transition the antiferromagnetic phase is metallic or not remains open. As in the case of $\kappa_{\text {av }}$, one would have to go to temperature $T \lesssim 10^{-2}$ to be able to decide whether the ground state of the disordered system with AFLRO is metallic or insulating. To summarize visually the results of our investigation, we nevertheless tried to construct a schematic ground state phase diagram of the Anderson-Hubbard model in $d=\infty$ by extrapolating our finite-temperature data to $T=0$. In Fig. 17, the phase 

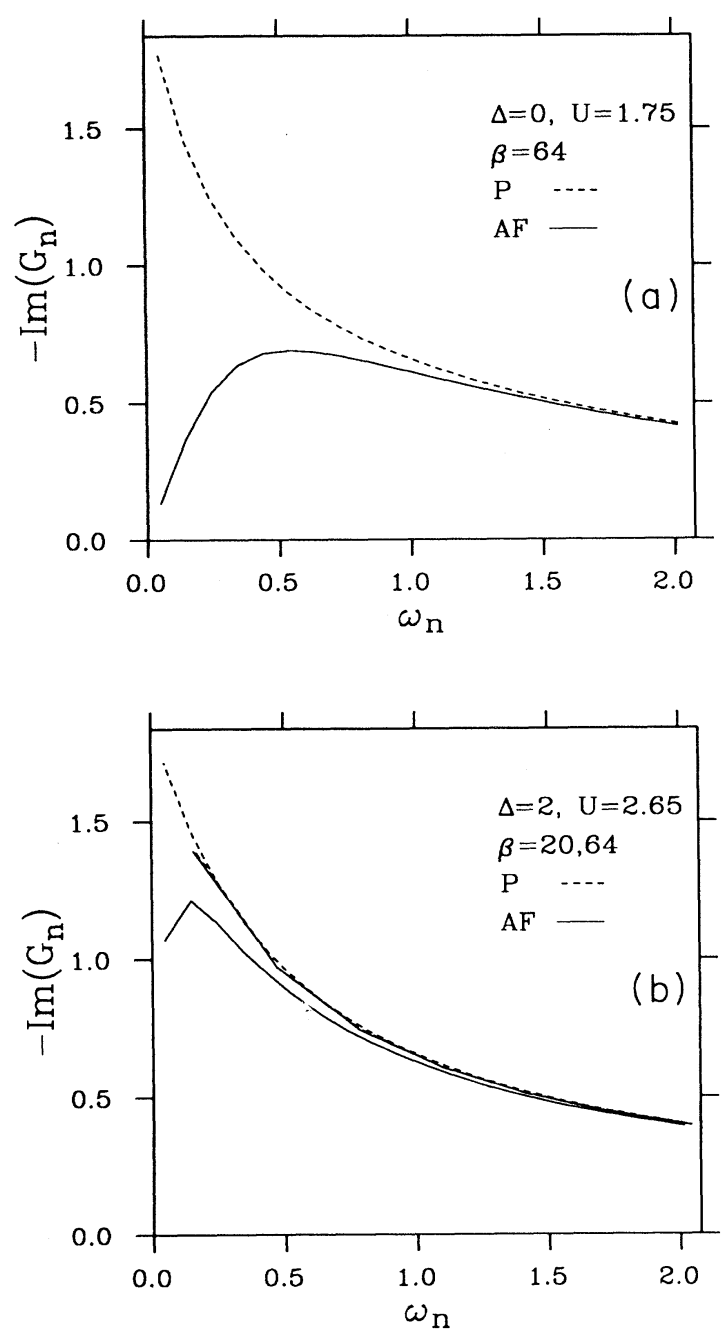

FIG. 15. Imaginary part of $G_{n}$ vs $\omega_{n}$ in the $P$ and AF phase; (a) no disorder, $U=1.75, \beta=64$; (b) binary alloy with $\Delta=2, U=2.65, \beta=20,64$ (the two curves at $\beta=20$ in the middle are undistinguishable).

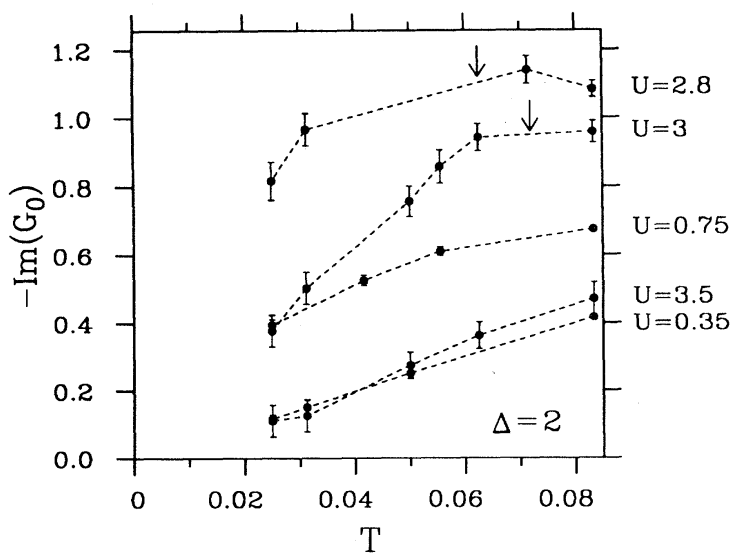

FIG. 16. Imaginary part of $G_{0}$ vs $T$ for the binary alloy with $\Delta=2$ for several values of $U$.

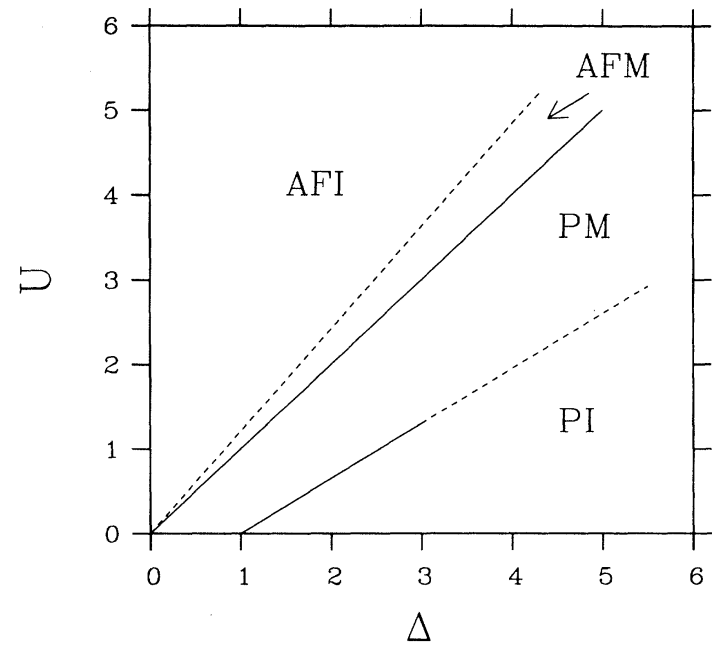

FIG. 17. Schematic phase diagram of the Anderson-Hubbard model in $d=\infty$ for a binary-alloy distribution. Dashed lines indicate transitions whose existence at $T=0$ has not yet been firmly established. (PI: paramagnetic insulator; PM: paramagnetic metal; AFM: antiferromagnetic metal; AFI: antiferromagnetic insulator.)

diagram in the case of the binary-alloy distribution is shown. For the continuous disorder distribution, (26b), the phase diagram is quite similar, but there is no paramagnetic insulating (PI) phase since disorder-induced band splitting does not occur for any value of $\Delta$. [The transition line between the PI phase and the paramagnetic metal $(\mathrm{PM})$ is effectively shifted to $\Delta=\infty$.]

\section{CONCLUSIONS}

In this paper, we presented a detailed, quantitative study of the physical effects caused by the simultaneous presence of interactions and randomness in a system of lattice electrons. To this end we investigated the Anderson-Hubbard model with diagonal disorder at half filling in the limit of infinite spatial dimensions, i.e., within a dynamical mean-field theory, for three different disorder distributions. Numerical results were obtained by employing quantum Monte Carlo techniques that provide an explicit finite-temperature solution of the model in $d=\infty$. No further approximations were used.

To construct the thermodynamic phase diagram, we derived and evaluated the appropriate averaged twoparticle correlation function, i.e., a dynamical response function, whose poles determine the magnetic instabilities of the disordered, interacting system. Only this function - and not the averaged staggered susceptibility $\chi_{\mathrm{AF}}$ itself, which is only a weighted sum of the response function over the (Matsubara) frequencies - fulfills a closed equation that determines the two-particle spectrum. The value of the response function at the lowest frequency is the most sensitive indicator for an instability of the system. In the temperature dependence of $\chi_{\mathrm{AF}}$, two distinct disorder regimes are observed: (i) for 
weak disorder, the Curie-Weiss law holds, while (ii) at strong disorder, $\chi_{\mathrm{AF}}$ acquires a maximum at a temperature below which a crossover from the temperature- to the disorder-dominated regime takes place.

We demonstrated that at low temperatures and sufficiently strong interaction there always exists a phase with antiferromagnetic long-range order (AFLRO). Furthermore, we discovered a new strong-coupling anomaly, namely, that the Néel-temperature $T_{N}$ is not always a monotonously decreasing function of disorder. Indeed, at strong coupling and not too large disorder $T_{N}$ is always found to be an increasing function of disorder, i.e., disorder favors the formation of AFLRO in this regime. This implies the existence of an unexpected disorder-induced transition to a phase with AFLRO. Under the assumption that $T_{N}$ is proportional to the effective exchange coupling between spins even in the disordered system, we proved that for diagonal disorder the anomalous behavior is generic, i.e., is independent of the type of disorder distribution. It is a consequence of the fact that for diagonal disorder the difference between the local energies of neighboring sites becomes smaller on average, thus leading to stronger effective exchange coupling.

We then studied the existence of metal-insulator transitions in the Anderson-Hubbard model. Although in $d=\infty$ Anderson localization does not take place, the presence of disorder may well have other strong effects. In particular, binary-alloy disorder is able to cause band splitting (thereby resembling the effect of genuine interactions) and hence may induce a metal-insulator transition all by itself. Special attention was given to the question of whether or not the disorder allows for the stabilization of an antiferromagnetic (AF) metal. To this end, the average compressibility $\kappa_{\text {av }}$ was evaluated both in the paramagnetic $(P)$ and AF phase. Contrary to our expectation, the presence of disorder was found to enhance the metallicity of the AF phase close to the $P$ -
AF transition. This enhancement strongly suggests the existence of an AF metal in the low-temperature phase of the Anderson-Hubbard mode. To investigate whether this AF phase persists to be metallic down to $T=0$, at least in $d=\infty$, we studied the frequency components of the one-particle Green function and of $\kappa_{\text {av }}$, respectively, down to $T=1 / 64$, our lowest temperature. However, a reliable answer to this question can only be found at still lower temperatures, which at present are beyond the reach of the finite-temperature Monte Carlo techniques used here. In any case, the transition scenario involving metal-insulator and $P$-AF transitions obtained for the Anderson-Hubbard model is remarkably rich. For alloytype disorder with $\Delta>1$ (split-band limit for $U=0$ ) at $T=0$, an increase of the interaction $U$ from zero will probably first lead to a transition from a paramagnetic insulator to a paramagnetic metal at $U_{c}^{M I, 1}$, then, at $U_{c}^{\mathrm{AF}}$, to an antiferromagnetic metal and finally, at $U_{c}^{M I, 2}$, to an antiferromagnetic insulator. No compelling evidence was found that $U_{c}^{\mathrm{AF}}$ and $U_{c}^{M I, 2}$ coincide. In the case of a continuous disorder distribution, one has $U_{c}^{M I, 1}=0$ since band splitting never occurs.

The above findings prove that the interplay between electronic interactions and scattering from disorder leads to interesting physical effects. In particular, the strong coupling anomaly discovered here calls for an experimental verification.

\section{ACKNOWLEDGMENTS}

We are grateful to A. Altland, P. van Dongen, M. Jarrell, H. Müller-Krumbhaar, and especially E. Müller-Hartmann for very useful discussions and comments. This work was supported in part by the Sonderforschungsbereich 341 of the Deutsche Forschungsgemeinschaft.
* Present address: Physics Department, University of California, Davis, CA 95616.

${ }^{\dagger}$ Permanent address: Institute of Physics, Academy of Sciences of the Czech Republic, CZ-18040 Praha 8, Czech Republic.

${ }^{1}$ F. J. Wegner, Z. Phys. B 35, 207 (1979).

${ }^{2}$ E. Abrahams, P. W. Anderson, G. Licciardello, and T. V. Ramakrishnan, Phys. Rev. Lett 42, 673 (1979).

${ }^{3}$ For a review, see, P. A. Lee and T. V. Ramakrishnan, Rev. Mod. Phys. 57, 287 (1985).

${ }^{4}$ A. M. Finkelshtein, Zh. Eksp. Teor. Fiz. 84, 168 (1983) [Sov. Phys. JETP 57, 97 (1983)].

${ }^{5}$ C. Castellani, C. Di Castro, P. A. Lee, and M. Ma, Phys. Rev. B 30, 527 (1984); C. Castellani, C. Di Castro, and M. Grilli, ibid. 34, 5907 (1986).

${ }^{6}$ A. M. Finkelshtein, Z. Phys. B 56, 189 (1984).

${ }^{7}$ C. Castellani, C. Di Castro, P. A. Lee, M. Ma, S. Sorella, and E. Tabet, Phys. Rev. B 30, 1596 (1984); 33, 6169 (1986).

${ }^{8}$ C. Castellani, G. Kotliar, and P. A. Lee, Phys. Rev. Lett. 59, 323 (1987).
${ }^{9}$ D. Belitz and T. R. Kirkpatrick, Rev. Mod. Phys. 66, 261 (1994).

${ }^{10}$ M. Milovanović, S. Sachdev, and R. N. Bhatt, Phys. Rev. Lett. 63, 82 (1989).

${ }^{11}$ A. Langenfeld and P. Wölfle, Ann. Phys. (Leipzig) 4, 43 (1995).

${ }^{12}$ M. Ma, Phys. Rev. B 26, 5097 (1982).

${ }^{13}$ J. Yi, L. Zhang, and G. S. Canright, Phys. Rev. B 49, 15920 (1994).

${ }^{14}$ G. T. Zimanyi and E. Abrahams, Phys. Rev. Lett. 64, 2719 (1990).

${ }^{15}$ R. Shankar, Int. J. Mod. Phys. B 4, 2371 (1990).

${ }^{16}$ H. Pang, S. Liang, and J. F. Annett, Phys. Rev. Lett. 71, 4377 (1994).

${ }^{17}$ W. Metzner and D. Vollhardt, Phys. Rev. Lett. 62, 324 (1989).

${ }^{18}$ For a review, see D. Vollhardt, in Correlated Electron Systems, edited by V. J. Emery (World Scientific, Singapore, $1993)$, p. 57.

${ }^{19}$ E. Müller-Hartmann, Z. Phys. B 74, 507 (1989); 76, 211 (1989). 
${ }^{20}$ V. Janiš, Z. Phys. B 83, 227 (1991).

${ }^{21}$ A. Georges and G. Kotliar, Phys. Rev. B 45, 6479 (1992).

${ }^{22}$ V. Janiš and D. Vollhardt, Int. J. Mod. Phys. B 6, 731 (1992).

${ }^{23}$ A. Georges, G. Kotliar, and Q. Si, Int. J. Mod. Phys. B 6, 705 (1992).

${ }^{24}$ M. Jarrell, Phys. Rev. Lett. 69, 168 (1992).

${ }^{25}$ M. Jarrell and T. Pruschke, Z. Phys. B 90, 187 (1993).

${ }^{26}$ In fact, in their exact solution of the Falicov-Kimball model in $d=\infty$, Brandt and Mielsch [Z. Phys. B 75, 365 (1989); $\mathbf{7 9}, 295$ (1990); 82, 37 (1991)] were the first to show that the local self-energy may be used to map the lattice problem in $d=\infty$ onto its atomic counterpart with an additional "Kadanoff-Baym field," which has to be determined self-consistently; for an application to other Hubbard-like models, see G. Hülsenbeck and F. Stephan, Z. Phys. B 94, 281 (1994).

${ }^{27}$ See, also, F. J. Ohkawa, J. Phys. Soc. Jpn. 60, 3218 (1991); 61, 1615 (1991).

${ }^{28}$ J. E. Hirsch and R. M. Fye, Phys. Rev. Lett. 56, 2521 (1986).

${ }^{29}$ A. Georges and W. Krauth, Phys. Rev. B 48, 7167 (1993).

${ }^{30} \mathrm{M}$. J. Rozenberg, X. Y. Zhang, and G. Kotliar, Phys. Rev. Lett. 69, 1236 (1992).

${ }^{31}$ A. Georges and W. Krauth, Phys. Rev. Lett. 69, 1240 (1992).

32 X. Y. Zhang, M. J. Rozenberg, and G. Kotliar, Phys. Rev. Lett. 70, 1666 (1993); M. J. Rozenberg, G. Kotliar, and X.Y. Zhang, Phys. Rev. B 49, 10181 (1994); Q. Si, M. J. Rozenberg, G. Kotliar, and A. E. Ruckenstein, Phys. Rev. Lett. 72, 2761 (1994).

${ }^{33}$ T. Pruschke, D. L. Cox, and M. Jarrell, Phys. Rev. B 47, 3553 (1993); M. Jarrell and T. Pruschke, ibid. 49, 1458 (1994).

${ }^{34}$ A. Georges, G. Kotliar, and W. Krauth, Z. Phys. B 92, 313 (1993).

${ }^{35}$ M. Jarrell, H. Akhlaghpour, and T. Pruschke, Phys. Rev. Lett. 70, 1670 (1993).

${ }^{36}$ J. K. Freericks, M. Jarrell, and D. J. Scalapino, Phys. Rev. B 48, 6302 (1993).

${ }^{37}$ A. Khurana, Phys. Rev. Lett. 64, C1990 (1990).

${ }^{38}$ R. Vlaming and D. Vollhardt, Phys. Rev. B 45, 4637 (1992).

${ }^{39}$ V. Janiš and D. Vollhardt, Phys. Rev. B 46, 15172 (1992).

${ }^{40}$ B. Velický, S. Kirkpatrick, and H. Ehrenreich, Phys. Rev. 175, 747 (1968).

${ }^{41}$ J. M. Ziman, Models of Disorder (Cambridge University Press, Cambridge, 1979)

${ }^{42}$ V. Janiš, M. Ulmke, and D. Vollhardt, Europhys. Lett. 24,
287 (1993).

${ }^{43}$ V. Dobrosavljević and G. Kotliar, Phys. Rev. Lett. 71, 3218 (1993); Phys. Rev. B 50, 1430 (1994).

${ }^{44}$ R. Vlaming, G. S. Uhrig, and D. Vollhardt, J. Phys. Condens. Matter 4, 7773 (1992); 4, 10103 (1992); G. S. Uhrig and R. Vlaming, ibid. 5, 2561 (1993).

${ }^{45}$ G. S. Uhrig and R. Vlaming, Phys. Rev. Lett. 71, 271 (1993).

${ }^{46} \mathrm{G}$. S. Uhrig and D. Vollhardt (unpublished).

${ }^{47}$ In principle, the grand potential (6) may equally be viewed as a functional of the set of complex functions $\Sigma_{\sigma n}$ only in which case, however, one has to supplement (6) by the definition of $G_{\sigma n}$ as the local (i.e., site-diagonal) element of the full temperature Green function (see below). In the initial formulation of the theory it is advisable to treat both sets of Legendre-conjugate variables $\Sigma_{\sigma n}$ and $G_{\sigma n}$ as variational parameters, since only then does the expression (6) contain the full information about the thermodynamic equilibrium of the system (Ref. 22). An equilibrium corresponds to a saddle point where the variations of $\Omega_{\mathrm{av}}$ with respect to $\Sigma_{\sigma n}$ and $G_{\sigma n}$ vanish, i.e., $\delta \Omega_{\mathrm{av}} / \delta G_{\sigma n}=0$ and $\delta \Omega_{\mathrm{av}} / \delta \Sigma_{\sigma n}=0$. These variational conditions determine the physical values of $G_{\sigma n}$ and $\Sigma_{\sigma n}$ as the local Green function and self-energy of the electrons, respectively. A theory generated in this way is guaranteed to be thermodynamically consistent and conserving in the spirit of the Baym-Kadanoff formalism [G. Baym, Phys. Rev. 127, 836 (1962)].

${ }^{48}$ In the case of a broken symmetry, one needs to introduce more than one local effective potential [see Eq. (10)].

${ }^{49}$ Here, we consider $\Omega_{\mathrm{av}}$ as a functional of $\Sigma_{\sigma n}$ only, i.e., we assume that we already established the identity of $G_{\sigma n}$ as the local Green function (see the discussion in Ref. 47).

${ }^{50}$ Note that the energy at site $i$ is measured relative to the surrounding medium; that is why the effective local propagator of electrons at site $i$ is given by $\mathcal{G}_{\sigma n}=\left[G_{\sigma n}^{-1}+\Sigma_{\sigma n}\right]^{-1}$.

${ }^{51}$ V. Janiš, Czech. J. Phys. B 36, 1107 (1986); Phys. Rev. B 40, 11331 (1989).

${ }^{52}$ W. H. Press, B. P. Flannery, S. A. Teukolsky, and W. T. Vetterling, Numerical Recipes: The Art of Scientific Computing (Cambridge University Press, Cambridge, 1988).

${ }^{53}$ All calculations were performed on a Cray YMP of the Forschungszentrum Jülich.

${ }^{54}$ This was pointed out to us for the binary-alloy case by $\mathrm{E}$. Müller-Hartmann.

${ }^{55}$ Fractals in Disordered Systems, edited by A. Bunde and S. Havlin (Springer, Berlin, 1991).

${ }^{56}$ D. Mattis (unpublished), as cited by E. H. Lieb and F. Y. Wu, Phys. Rev. Lett. 20, 1445 (1968). 